INT \#DOE/ER/40561-245-INT96-00-114

\title{
Chiral Symmetry and the Nucleon's Vector Strangeness Form Factors
}

\author{
M.J. Musolf ${ }^{a, b *}$ and Hiroshi Ito $^{c}$ \\ ${ }^{a}$ Continuous Electron Beam Accelerator Facility Newport News, VA 23606 USA \\ ${ }^{b}$ Institute for Nuclear Theory, University of Washington, Seattle, WA 98195 USA \\ ${ }^{c}$ Center for Nuclear Studies, Department of Physics, George Washington University \\ Washington, D.C. 20052 USA
}

\begin{abstract}
The nucleon's strange-quark vector current form factors are studied from the perspective of chiral symmetry. It is argued that chiral perturbation theory cannot yield a prediction for the strangeness radius and magnetic moment. Arrival at definite predictions requires the introduction of additional, modeldependent assumptions which go beyond the framework of chiral perturbation theory. A variety of such model predictions is surveyed, and the credibility of each is evaluated. The most plausible prediction appears in a model where the unknown chiral counterterms are identified with $t$-channel vector meson exchange amplitudes. The corresponding prediction for the mean square Dirac strangeness radius is $\left\langle r_{s}^{2}\right\rangle=0.24 \mathrm{fm}^{2}$, which would be observable in up-coming semileptonic determinations of the nucleon's strangeness form factors.
\end{abstract}

11.30.Rd, 12.40.-y, 14.20.Dh

Typeset using REVTEX

*On leave from the Department of Physics, Old Dominion University, Norfolk, VA 23529 USA 


\section{INTRODUCTION}

There has been considerable interest recently in the strange quark "content" of the nucleon [1-15]. The reasons for this interest are both theoretical and phenomenological. In the latter case, early analyses of the pion-nucleon sigma term [26] and later results for the nucleon's inclusive, spin-dependent deep-inelastic structure functions [27 31] suggested that a non-trivial fraction of the nucleon's mass and spin are carried by the $s \bar{s}$ component of the sea. Subsequent analyses of the sigma-term have reduced the value of $\langle p|\bar{s} s| p\rangle /\langle p|\bar{u} u+\bar{d} d| p\rangle$, and therefore the strange-quark contribution to $m_{N}$, by a factor of two [32], while studies of $\mathrm{SU}(3)$-breaking in the axial vector octet imply a theoretical uncertainty in the value of $\Delta s$, the strange quark contribution to the nucleon's spin extracted from deep inelastic scattering (DIS) measurements, sufficiently large to make the bounds on $\Delta s$ consistent with zero [33 36]. Nevertheless, the early analyses of the sigma term and polarized DIS results have motivated proposals to measure another strange-quark observable, $\left\langle p\left|\bar{s} \gamma_{\mu} s\right| p\right\rangle$. Indeed, several low- and medium-energy parity-violating electron scattering experiments are either underway or planned at MIT-Bates [3, 3], CEBAF [4 [6] and Mainz [7] with the goal of measuring the two form factors which parameterize the nucleon's strange quark vector current, $G_{E}^{(s)}$ and $G_{M}^{(s)}$.

Theoretically, strange-quarks are interesting because they don't appear explicitly in most quark model descriptions of the nucleon. Although the quark model provides a useful intuitive picture of the nucleon's substructure and has seen considerable success in accounting for a wide range of properties of the low-lying hadrons [37], one knows that there is more to the nucleon than the three constituent quarks. In particular, processes such as DIS and Drell-Yan have provided considerable insight regarding the important role played by the $q \bar{q}$ and gluon sea when the nucleon interacts at high energies [8]. Almost no information exists, however, regarding the low-energy manifestations of the sea. Because strange quarks constitute purely sea degrees of freedom, low- and intermediate-energy determinations of strange quark matrix elements offer a new window on the "low-energy" structure of the nucleon which goes beyond the description provided by the quark model. In particular, the weak neutral current scattering experiments mentioned above should set bounds on the spatial polarization of the $s \bar{s}$ sea [4,6], its contributions to the nucleon magnetic moment [2 [4] and spin [9], and its role in the nuclear response at moderate momentum transfer [5].

One has seen considerable progress over the past few years in clarifying the interpretation of neutral current observables in terms of strangeness matrix elements [1, 10, 13]. The situation regarding theoretical predictions for these matrix elements is less advanced. Ideally, one would hope to draw inferences from the deep inelastic data on $s$ - and $\bar{s}$-distributions [38] for elastic vector and axial vector strangeness matrix elements. However, the highenergy data provide light-cone momentum distribution functions, and one does not know at present how to translate this information into the spin and spatial nucleon wavefunctions as needed to compute charge radii, magnetic moments, etc. 39. Similarly, one might hope for first-principles microscopic predictions using lattice QCD. To date, lattice results for the strangeness axial charge [14] and strangeness magnetic moment [15] have been obtained in the quenched approximation, and one anticipates a refinement of these results as lattice methods continue to advance. In the absence of definitive lattice calculations - and with an 
eye toward understanding the mechanisms which govern the scale of nucleon strangeness - a variety of model calculations have been performed. The latter have yielded a wide array of predictions for strangeness matrix elements which vary in both magnitude and sign 1625. While one might argue ad nauseum about the relative merits of different models, there is no compelling reason to take any particular model calculation as definitive.

In an effort to add some clarity to this situation, we discuss in this paper the implications for nucleon strangeness vector current matrix elements of one of the underlying, approximate symmetries of QCD: chiral symmetry. The use of chiral symmetry, in the guise of chiral perturbation theory (CHPT), has proven highly effective in predicting and interpreting a wide variety of low-energy observables [40,41]. The essential strategy of CHPT is to exploit the approximate $\mathrm{SU}(3)_{L} \times \mathrm{SU}(3)_{R}$ chiral symmetry of QCD for the three lightest flavors to relate one set of observables to another (accounting for loop effects), or to draw on one set of measured quantities to predict another. This approach has recently been employed to analyze of baryon octet and decuplet magnetic moments [42 44] and the nucleon's isovector charge radius 455. As we illustrate below, this strategy breaks down in the flavor-singlet channel, rendering CHPT un-predictive for the nucleon's strangeness matrix elements. The reason is that the coefficients of the relevant flavor-singlet operators in the chiral Lagrangian, which contain information on short-distance hadronic effects, cannot be determined from existing measurements by using chiral symmetry. Although the leading, non-analytic long-distance (loop) contributions are calcuable $\left(\mathcal{O}\left(\sqrt{m_{s}}\right)\right.$ for the strangeness magnetic moment and $\mathcal{O}\left(\ln m_{s}\right)$ for the strangeness radius), one has no reason to assume that they are numerically more important than the unknown analytic terms arising at the same or lower order from the chiral Lagrangian. The only rigorous way to determine these unknown analytic contributions is to measure the very quantity one would like to predict: the nucleon's flavor-singlet current matrix element.

Consequently, if one wishes to make any predictions at all, one must invoke additional - and therefore model-dependent - assumptions. We illustrate this next line of defense in three forms: (a) a "resonance saturation" model in which the unknown constants arising in chiral perturbation theory are determined by the $t$-channel exchange of vector mesons; (b) a class of models in which the nucleon's "kaon cloud" is assumed to dominate the strangeness form factors; and (c) constituent chiral quark models in which nucleon's strangeness matrix elements arise from the strangeness content of the constituent $U$ - and $D$-quarks. For each of these approaches, we present new calculations and compare them with calculations discussed elsewhere in the literature. The corresponding results are unabashedly model-dependent and, therefore, not strong. We give them mainly to illustrate the outer limits to which one might go in employing chiral symmetry to compute $G_{E}^{(s)}$ and $G_{M}^{(s)}$. Although there exist additional chiral model approaches not considered in detail here, we believe that the three which we discuss are sufficiently representative so as to illustrate the breadth of predictions permitted by chiral symmetry. Among these predictions, there does appear to be one having a greater degree of credibility than others: the value of the Dirac mean square strangeness radius arising from the vector meson exchange, or resonance saturation, model. Nevertheless, even in this case, the logic is not airtight. A more detailed analysis of the strangeness and isoscalar electromagnetic form factors within the framework of dispersion relations could reveal important contributions not included in the resonance saturation approach.

We organize our discussion of these points as follows. In Section II we review the ef- 
fective low-energy chiral Lagrangians which describe the interaction of pseudoscalar mesons with baryons or quarks. In Section III we employ this formalism to compute the nucleon's strange-quark vector current form factors, introducing model assumptions as necessary and evaluating their credibility. Section IV gives the results of these calculations and a discussion of their meaningfulness. Section V summarizes our conclusions.

\section{CHIRAL LAGRANGIANS}

In the low-energy world of three-flavor QCD, the QCD Lagrangian manifests an approximate $\mathrm{SU}(3)_{L} \times \mathrm{SU}(3)_{R}$ chiral symmetry. This symmetry is explicitly broken by the small current quark masses. In addition, spontaneous symmetry-breaking $\mathrm{SU}(3)_{L} \times \mathrm{SU}(3)_{R} \rightarrow \mathrm{SU}(3)_{V}$ implies the existence of eight massless (assuming $m_{q}=0$ ) Goldstone modes and an axial vector condensate. One identifies the latter with the pion decay constant $f_{\pi} \approx 93 \mathrm{MeV}$ and the former with the lowest-lying octet of pseudoscalar mesons. The Goldstone bosons are conveniently described by a field $\Sigma$, given by

$$
\Sigma=\exp [2 i \tilde{\Pi} / f]
$$

where $f \equiv f_{\pi}$ and

$$
\tilde{\Pi}=\frac{1}{2} \sum_{a=1}^{8} \lambda_{a} \phi_{a}
$$

with the $\lambda_{a}$ being the eight Gell-Mann matrices and the $\phi_{a}$ being the pseudoscalar meson fields 46,47. The Lagrangian which describes the pseudoscalar kinetic energies and self interactions is given by

$$
\mathcal{L}=\frac{f^{2}}{4} \operatorname{Tr}\left(\partial^{\mu} \Sigma^{\dagger} \partial_{\mu} \Sigma\right)+\frac{f^{2}}{2}[\operatorname{Tr}(\Sigma \mu M)+\text { h.c. }]
$$

where $M=\operatorname{diag}\left[m_{u}, m_{d}, m_{s}\right]$ is just the QCD current quark mass matrix which explicitly breaks the residual $\mathrm{SU}(3)_{V}$ symmetry and $\mu$ is a parameter which relates the quark masses to quadratic forms in the pseudscalar masses (hence, $m_{\pi, K}$ is of order $\sqrt{m_{q}}$ ). The Lagrangian in Eq. (3) actually constitutes the leading term in an expansion in powers of $p / \Lambda_{\chi}$ and $\mu M / \Lambda_{\chi}$, where $p$ denotes the momentum of a low-energy pseudoscalar meson and $\Lambda_{\chi} \approx 4 \pi f \approx 1$ $\mathrm{GeV}$ is the scale of chiral symmetry breaking. For purposes of the present study, retention of higher-order terms in the chiral expansion of the purely mesonic sector is not necessary.

Interactions between the Goldstone bosons and matter fields are conveniently described by first introducing vector and axial vector currents

$$
\begin{aligned}
V_{\mu} & \equiv \frac{1}{2}\left(\xi^{\dagger} \partial_{\mu} \xi+\xi \partial_{\mu} \xi^{\dagger}\right) \\
A_{\mu} & \equiv \frac{i}{2}\left(\xi^{\dagger} \partial_{\mu} \xi-\xi \partial_{\mu} \xi^{\dagger}\right)
\end{aligned}
$$

where $\Sigma=\xi^{2}$. One may now proceed to construct a chiral Lagrangian for fermions. The simplest case involves the effective, consituent quarks of the quark model. Letting 


$$
\psi=\left(\begin{array}{c}
U \\
D \\
S
\end{array}\right)
$$

denote the triplet of light-quark fields, one has for the leading term in the chiral expansion

$$
\mathcal{L}_{Q}=\bar{\psi}(i \not D-m) \psi+g_{A} \bar{\psi} A \gamma_{5} \psi
$$

where

$$
D_{\mu} \equiv \partial_{\mu}+V_{\mu}
$$

is a chiral covariant derivative and $g_{A}$ is a constant which governs the strength of the interaction between quarks and odd numbers of pseudoscalar mesons (the last term in Eq. (7)). The term involving $m$ gives the constituent quark masses" in the limit of good $\mathrm{SU}(3)_{V}$ symmetry. Higher-order terms in the chiral expansion include those which break the degeneracy between the constituent quarks. In the chiral quark model calculation we discuss below, we allow for mass splittings among the constituent quarks, although we will not show the $\mathrm{SU}(3)_{V}$ symmetry-breaking terms explicitly. The higher order terms in the chiral expansion relevant to strangeness vector current matrix elements will be introduced below.

In the case of meson-baryon interactions, more thought is required when writing down an effective chiral Lagrangian. In the most naïve approach, one assigns baryons to the appropriate $\mathrm{SU}(3)$ multiplet and constructs objects using this multiplet, derivatives, $V_{\mu}$ and $A_{\mu}$ which transform as $\mathrm{SU}(3)_{L} \times \mathrm{SU}(3)_{R}$ singlets. For the lowest-lying octet of baryons, one has the matrix representation

$$
B \equiv \frac{1}{\sqrt{2}} \sum_{a=1}^{8} \lambda_{a} \psi_{a}
$$

where the $\psi_{a}$ are the octet baryon fields. The naïve, leading-order baryon Lagrangian is, then,

$$
\mathcal{L}_{B}=\operatorname{Tr}\left[\bar{B}\left(i \not D-m_{B}\right) B\right]+D \operatorname{Tr}\left(\bar{B} \gamma_{\mu} \gamma_{5}\left\{A^{\mu}, B\right\}\right)+F \operatorname{Tr}\left(\bar{B} \gamma_{\mu} \gamma_{5}\left[A^{\mu}, B\right]\right),
$$

where, in this case, the action of the chiral covariant derivative on the baryon fields is given by

$$
D^{\mu} B=\partial^{\mu} B+\left[V^{\mu}, B\right],
$$

and where $m_{B}$ gives the octet baryon masses in the limit of good $\mathrm{SU}(3)_{V}$ symmetry. The constants $D$ and $F$ are the usual SU(3) reduced matrix elements.

As in the case of the quark chiral Lagrangian, one could formally write down corrections to $\mathcal{L}_{B}$ as a series in powers of $p / \Lambda_{\chi}$ and $m_{B} / \Lambda_{\chi}$. Such an expansion would not be convergent, however, since numerically one has $m_{B} \sim \Lambda_{\chi}$. This situation contrasts with that of chiral

\footnotetext{
${ }^{1}$ not to be confused with the current quark mass matrix $M$
} 
quarks whose constituent masses are well below the scale of chiral symmetry breaking. Consequently, one has no reason to believe that higher-order terms in the chiral expansion are less important than the terms given in Eq. (10). Jenkins and Manohar [33] developed an approach to circumvent this difficutly with the baryon chiral Lagrangian. The idea is to approximate the baryons as very heavy static fields whose spectrum is characterized by states of good velocity, $v_{\mu}=p_{\mu} / m_{B}$. One correspondingly redefines the baryon fields by rotating away the frequency dependence associated with the heavy mass:

$$
B_{v}(x) \equiv \exp \left(i m_{B} \not v \cdot x\right) B(x)
$$

The new baryon Lagrangian, specified now for baryons of given velocity, has a similar form to that appearing in Eq. (10) but with no baryon mass term. As a result, one can now perform a chiral expansion in powers of external momenta and baryon mass splittings divided by $\Lambda_{\chi}$ without encountering the problematic $m_{B} / \Lambda_{\chi}$ terms. Before writing this Lagrangian, we follow Ref. [33] and make use of the spin operators $S_{v}^{\lambda}$ which satisfy the relations

$$
\begin{aligned}
& v \cdot S=0 ; \quad S_{v}^{2} B_{v}=-(3 / 4) B_{v} ; \quad\left\{S_{v}^{\alpha}, S_{v}^{\beta}\right\}=(1 / 2)\left(v^{\alpha} v^{\beta}-g^{\alpha \beta}\right) ; \\
& {\left[S_{v}^{\alpha}, S_{v}^{\beta}\right]=i \epsilon^{\alpha \beta \mu \nu} v_{\mu} S_{v \nu} .}
\end{aligned}
$$

In addition, we use the relations

$$
\bar{B}_{v} \gamma^{\mu} B_{v}=v^{\mu} \bar{B}_{v} B_{v} ; \quad \bar{B}_{v} \gamma^{\mu} \gamma_{5} B_{v}=2 \bar{B}_{v} S_{v}^{\mu} B_{v} ; \quad \bar{B}_{v} \sigma^{\mu \nu} B_{v}=2 \epsilon^{\mu \nu \alpha \beta} v_{\alpha} \bar{B}_{v} S_{v \beta} B_{v} .
$$

Using these identities, the leading-order heavy baryon chiral Lagrangian is

$$
\mathcal{L}_{v}=i \operatorname{Tr}\left(\bar{B}_{v} v \cdot D B_{v}\right)+2 D \operatorname{Tr}\left(\bar{B}_{v} S_{v}^{\mu}\left\{A_{\mu}, B_{v}\right\}\right)+2 F \operatorname{Tr}\left(\bar{B}_{v} S_{v}^{\mu}\left[A_{\mu}, B_{v}\right]\right) .
$$

The first corrections to $\mathcal{L}_{v}$ contain one more power of the chiral covariant derivative, quark mass matrix $M$, or axial vector $A_{\mu}$.

In what follows, we compute strange quark vector currents of non-strange chiral quarks and non-strange baryons arising from kaon loops. To that end, it is useful to work instead with the baryon number current $J_{\mu}^{B}$ and to introduce a vector current source $Z^{\mu}$ which couples to $J_{\mu}^{B}$ via the minimal substitution $\partial^{\mu} \rightarrow \partial^{\mu}+i \hat{Q}_{B} Z^{\mu}$, where $\hat{Q}_{B}$ is the baryon number operator.2 Taking the first functional derivative with respect to $Z^{\mu}$ of the generating functional yields $n$-point functions with a single $J_{\mu}^{B}$ insertion. The strange quark current is related in a straightforward manner to $J_{\mu}^{B}$ and the isoscalar EM current (see Eqs. (20) 24) below). In practice, it is simpler to compute the strangeness charge of each particle appearing in a Feynman diagram, insert the appropriate Lorentz structure for a vector current, and evaluate the resulting contribution to the strangeness matrix element. From a formal standpoint, however, the use of the baryon number current and of the source $Z^{\mu}$ provides an efficient means for keeping track of the flavor content and chiral order associated with higher moments (mean square radius, magnetic moment, etc.) of various currents.

\footnotetext{
${ }^{2}$ The full chiral structure of the charge operator is given in Ref. [42]. For the present purpose, the inclusion of the full structure is not necessary.
} 


\section{STRANGE QUARK MATRIX ELEMENTS}

With the formalism of Section II in hand, it is straightforward to compute nucleon matrix elements of the strange-quark vector current, $\left\langle p^{\prime}\left|\bar{s} \gamma_{\mu} s\right| p\right\rangle$. This matrix element can be parameterized in terms of two form factors, $F_{1}^{(s)}$ and $F_{2}^{(s)}$ :

$$
\left\langle p^{\prime}\left|\bar{s} \gamma_{\mu} s\right| p\right\rangle=\bar{u}\left(p^{\prime}\right)\left[F_{1}^{(s)} \gamma_{\mu}+i \frac{F_{2}^{(s)}}{2 m_{N}} \sigma_{\mu \nu} Q^{\nu}\right] u(p)
$$

where $u(p)$ denotes a nucleon spinor and $Q=p^{\prime}-p$ is the momentum transfer to the nucleon. When working in the heavy baryon formalism, the corresponding Lorentz structures are obtained from Eq. (16) by the use of the relations in Eq. (13). For on-shell nucleons, the form factors are functions of $Q^{2}=q_{0}^{2}-|\vec{q}|^{2}$, where $Q^{\mu}=\left(q_{0}, \vec{q}\right)$. In what follows, we work with the so-called Sachs electric and magnetic form factors [48], defined as

$$
\begin{aligned}
& G_{E}^{(s)}=F_{1}^{(s)}-\tau F_{2}^{(s)} \\
& G_{M}^{(s)}=F_{1}^{(s)}+F_{2}^{(s)}
\end{aligned}
$$

where $\tau \equiv-Q^{2} / 4 m_{N}^{2}$. At $Q^{2}=0$, the Sachs electric form factor gives the net strangeness of the nucleon, which is zero. At small momentum transfer, the scale of this form factor is governed by the first derivative with respect to $Q^{2}$, which defines the mean square "strangeness radius". We work with a dimensionless version of this quantity, $\rho_{S}^{s}$, defined as

$$
\rho_{S}^{s}=\left.\frac{d G_{E}^{(s)}}{d \tau}\right|_{\tau=0}=-\frac{2}{3} m_{N}^{2}\left\langle r_{s}^{2}\right\rangle^{S}
$$

where $\left\langle r_{s}^{2}\right\rangle^{S}$ is the dimensionful Sachs strangeness radius and where the superscript "S" denotes the Sachs, as distinct from the Dirac, radius. There exists no symmetry principle which constrains the strangeness magnetic moment, $G_{M}^{(s)}(0)=\mu^{s}$. Note that since $G_{E}^{(s)}(0)=$ 0 one has $\mu^{s}=\kappa^{s}$. In discussing the implications of chiral symmetry for $\left\langle p^{\prime}\left|\bar{s} \gamma_{\mu} s\right| p\right\rangle$, we will be concerned primarily with these two parameters, $\rho^{s}$ and $\mu^{s}$.

\section{A. Heavy Baryon Chiral Perturbation Theory}

In terms of chiral counting, the strangeness magnetic moment and radius, like the corresponding electromagnetic quantities, appear, respectively, as order $1 / \Lambda_{\chi}$ and $1 / \Lambda_{\chi}{ }^{2}$ corrections to the leading order heavy baryon Lagrangian given in Eq. (15). In discussing these corrections, it is convenient to rewrite the strangeness vector current in terms of the electromagnetic and baryon number currents:

$$
\begin{aligned}
J_{\mu}^{E M}(T=1) & =V_{\mu}^{(3)} \\
J_{\mu}^{E M}(T=0) & =(1 / \sqrt{3}) V_{\mu}^{(8)} \\
J_{\mu}^{B} & =V_{\mu}^{(0)},
\end{aligned}
$$

where the $T=1$ and $T=0$ designations indicate the isovector and isoscalar elecromagnetic currents, where "B" denotes the baryon number current, and where 


$$
V_{\mu}^{(a)}=\bar{q} \frac{\lambda^{a}}{2} \gamma_{\mu} q \quad q=\left(\begin{array}{l}
u \\
d \\
s
\end{array}\right)
$$

Here, the $\lambda^{a}, a=1, \ldots 8$ are the usual Gell-Mann matrices, $\lambda^{0}=\frac{2}{3} \mathbf{I}$, and $q$ gives the triplet of QCD quark fields. In terms of the currents in Eq. (20-22) one has

$$
\bar{s} \gamma_{\mu} s=J_{\mu}^{B}-2 J_{\mu}^{E M}(T=0)
$$

With these definitions one may write down the higher-order heavy baryon Lagrangians corresponding to the EM and baryon number magnetic moments and charge radii:

$$
\begin{aligned}
\Delta \mathcal{L}_{E M}^{T=1} & =\frac{e}{\Lambda_{\chi}} \epsilon_{\mu \nu \alpha \beta} v^{\alpha}\left\{b_{+} \operatorname{Tr}\left(\bar{B}_{v} S_{v}^{\beta}\left\{\lambda^{3}, B_{v}\right\}\right)+b_{-} \operatorname{Tr}\left(\bar{B}_{v} S_{v}^{\beta}\left[\lambda^{3}, B_{v}\right]\right)\right\} F^{\mu \nu} \\
& -\frac{e}{\Lambda_{\chi}^{2}}\left\{c_{+} \operatorname{Tr}\left(\bar{B}_{v}\left\{\lambda^{3}, B_{v}\right\}\right)+c_{-} \operatorname{Tr}\left(\bar{B}_{v}\left[\lambda^{3}, B_{v}\right]\right)\right\} v_{\mu} \partial_{\lambda} F^{\mu \lambda} \\
\Delta \mathcal{L}_{E M}^{T=0} & =\frac{e}{\Lambda_{\chi}} \frac{1}{\sqrt{3}} \epsilon_{\mu \nu \alpha \beta} v^{\alpha}\left\{b_{+} \operatorname{Tr}\left(\bar{B}_{v} S_{v}^{\beta}\left\{\lambda^{8}, B_{v}\right\}\right)+b_{-} \operatorname{Tr}\left(\bar{B}_{v} S_{v}^{\beta}\left[\lambda^{8}, B_{v}\right]\right)\right\} F^{\mu \nu} \\
& -\frac{e}{\Lambda_{\chi}^{2}} \frac{1}{\sqrt{3}}\left\{c_{+} \operatorname{Tr}\left(\bar{B}_{v}\left\{\lambda^{8}, B_{v}\right\}\right)+c_{-} \operatorname{Tr}\left(\bar{B}_{v}\left[\lambda^{8}, B_{v}\right]\right)\right\} v_{\mu} \partial_{\lambda} F^{\mu \lambda} \\
\Delta \mathcal{L}_{B} & =\frac{b_{0}}{\Lambda_{\chi}} \epsilon_{\mu \nu \alpha \beta} v^{\alpha} \operatorname{Tr}\left(\bar{B}_{v} S_{v}^{\beta} B_{v}\right) Z^{\mu \nu} \\
& -\frac{c_{0}}{\Lambda_{\chi}^{2}} \operatorname{Tr}\left(\bar{B}_{v} B_{v}\right) v_{\mu} \partial_{\lambda} Z^{\mu \lambda}
\end{aligned}
$$

where $F^{\mu \nu}$ is just the ordinary EM field strength tensor, $Z^{\mu \nu}$ is the analogous quantity involving the source $Z^{\mu}$ coupling to baryon number, and $e$ is the proton's EM charge. In each $\Delta \mathcal{L}$, the terms of order $1 / \Lambda_{\chi}$ contribute to the anomalous magnetic moment and those of order $1 / \Lambda_{\chi}^{2}$ enter the charge radius [49]. For a given baryon, the magnetic moment and mean square radius will contain a contribution from $\Delta \mathcal{L}$ and a contribution from loops (non-analytic in $m_{q}$ ), as in Fig. 1:

$$
\begin{aligned}
& \kappa^{a}=\kappa_{\text {LOOP }}^{a}+\left(\frac{2 m_{B}}{\Lambda_{\chi}}\right) b^{a} \\
& \rho_{D}^{a}=\rho_{\text {LOOP }}^{a}-\left(\frac{2 m_{B}}{\Lambda_{\chi}}\right)^{2} c^{a},
\end{aligned}
$$

where $\kappa^{a}=F_{2}^{(a)}(0)$ is the anomalous magnetic moment, "a" denotes the corresponding flavor channel ( $T=0,1, s, \mathrm{SU}(3)$ singlet), and the subscript "D" indicates the slope of the Dirac form factor $\left(F_{1}\right)$ at $\tau=0$. In the case of the EM moments, the quantities $b^{a}$ and $c^{a}$ contain appropriate linear combinations of $b_{ \pm}$and $c_{ \pm}$as determined from the traces appearing in Eqs. (25-26). Using the heavy baryon formalism outlined above, we compute $\kappa_{L O O P}^{a}$ and $\rho_{L O O P}^{a}$ employing dimensional regularization. For the strangeness moments, we find 


$$
\begin{aligned}
& \kappa_{\text {LOOP }}^{s}=(2 \pi)\left[\left(\frac{3 F+D}{\sqrt{6}}\right)^{2}+\frac{3}{2}(D-F)^{2}\right] \frac{m_{N}}{\Lambda_{\chi}} \frac{m_{K}}{\Lambda_{\chi}} \\
& \rho_{\text {LOOP }}^{s}=\left(\frac{m_{N}}{\Lambda_{\chi}}\right)^{2}\left\{1+\frac{5}{3}\left[\left(\frac{3 F+D}{\sqrt{6}}\right)^{2}+\frac{3}{2}(D-F)^{2}\right]\right\}\left[\mathcal{C}_{\infty}-\ln \frac{m_{K}^{2}}{\mu^{2}}\right],
\end{aligned}
$$

where $\mathcal{C}_{\infty}=1 / \varepsilon-\gamma+\ln 4 \pi$ with $\varepsilon=(4-d) / 2$ and $d$ being the number of dimensions. One finds analogous expressions for the isovector $\left(\lambda^{3}\right)$ and isoscalar $\left(\lambda^{8} / \sqrt{3}\right)$ components of the EM moments [42,50]. The constant $c^{s}$ appearing in Eq. (29) contains the appropriate dependence on $\mathcal{C}_{\infty}$ to cancel the pole term in $\rho_{L O O P}^{s}$. The scale $\mu$ denotes the scale at which the subtraction of the pole term is carried out. The remaining finite parts of $\left(\kappa_{L O O P}^{s}, \rho_{L O O P}^{s}\right)$ and of $\left(b^{s}, c^{s}\right)$ determine the value of the anomalous magnetic moment and mean square Dirac radius. Using Eqs. (2427), one can express the "low-energy" constants $\left(b^{s}, c^{s}\right)$ in terms of the corresponding quantities for the baryon and EM currents:

$$
\begin{aligned}
& b^{s}=b_{0}-2\left[b_{-}-\left(b_{+} / 3\right)\right] \\
& c^{s}=c_{0}-2\left[c_{-}-\left(c_{+} / 3\right)\right] .
\end{aligned}
$$

In the case of the EM moments, the $\left(b_{ \pm}, c_{ \pm}\right)$are fit to known EM moments in the baryon octet. One may then employ Eqs. (25-29) and the loop contributions to predict the moments of other baryons within the octet. This approach reflects the basic strategy of chiral perturbation theory: rely on chiral symmetry to relate one set of quantities (known EM moments) to another (those one wishes to predict), modulo loop corrections (a consequence of spontaneous chiral symmetry breaking). A simple fit to the nucleon EM moments alone gives $b_{+} \approx 1.4, b_{-} \approx 0.9, c_{+} \approx-1.9, c_{-} \approx 0.9$ [51].

As one would expect on general grounds, these constants are of order unity. In the case of the nucleon EM anomalous magnetic moments, the contributions from the $b_{ \pm}$and the loops have comparable magnitudes. In the case of the charge radii, the loops give the dominant contribution to the isovector EM charge radius while the $c_{ \pm}$give the dominant contribution to the isoscalar EM charge radius. It is evident, then, that one cannot rely on either the loop or the "counterterm" contributions alone to account for the nucleon's EM moments.

In the case of the strangeness magnetic moment and radius, one would ideally follow a similar strategy. However, the coefficients $b^{s}$ and $c^{s}$ are unknown. The reason is that these constants depend on $b_{0}$ and $c_{0}$ as well as the $b_{ \pm}$and $c_{ \pm}$. Since the baryon number magnetic moment and charge radius for the octet baryons have not be measured, $b_{0}$ and $c_{0}$ are un-determined. In fact, by virtue of Eq. (24), measurements of the strangeness radius and magnetic moment of the nucleon would provide a determination of the corresponding quantities for the baryon number current and, through Eqs. (32-33), would fix $b_{0}$ and $c_{0}$. Moreover, given the situation in the EM case, one would not be safe in assuming that $b^{s}$ and $c^{s}$ differ significantly in magnitude from unity. Indeed, one has no reason to expect, based on any symmetry principle, that either the loops or chiral counterterms should give

\footnotetext{
${ }^{3}$ Henceforth, the cancellation of the $\mathcal{C}_{\infty}$ will be understood and $\left(b^{a}, c^{a}\right)$ will denote the finite remainders of the counterterms.
} 
the dominant contributions to the strangeness radius and magnetic moment. Thus, chiral perturbation theory, in its purest form, cannot make a prediction for the strangeness vector current matrix elements.

In arriving at this conclusion, we did not include decuplet baryons in the loops nor the sub-leading non-analytic loop contributions $\left(\sqrt{m_{s}} \ln m_{s}\right.$ in the case of the strangeness magnetic moment) as was done in Ref. [42]. In that work, it was found that the dominant loop contribution to the magnetic moments is $\mathcal{O}\left(\sqrt{m_{s}}\right)$ and that the inclusion of the decuplet states does not have the same kind of effect as in the axial vector matrix elements, where non-negligible octet-decuplet cancellations occur for the loop contributions. Similarly, we did not use the one-loop corrected axial meson-nucleon couplings. Although from a formal standpoint the difference between tree-level and one-loop corrected couplings is of higher order than we are considering here, the authors of Ref. 42] obtained a better fit to the baryon magnetic moments with the corrected couplings. The use of the latter effectively reduces the size of the large kaon loop contributions. As we note below, the physics which modifies one loop results largerly amounts to kaon rescattering (see, e.g. Ref. [52]). Employing one-loop corrected axial couplings in the one-loop magnetic moment calculation incorporates some, but not all, rescattering contributions. It is not entirely clear that the impact of two-loop contributions to the magnetic moment is numerically less significant than the replacement of tree-level with one-loop corrected axial couplings in the one-loop magnetic moment calculation. In the present instance, we avoid this issue altogether and restrict our attention to one-loop effects.

\section{B. Chiral Models}

The conclusion of the foregoing analysis implies that in order to make predictions for the nucleon's strangeness moments, one must go beyond the framework of CHPT and invoke additional, model-dependent assumptions. To this end, a number of possibilities present themselves. We consider three such model-approaches: (a) resonance saturation, (b) kaon cloud dominance, and (c) constituent chiral quarks.

Resonance saturation. One could, for example, attempt to estimate the $b^{s}$ and $c^{s}$ by assuming that the corresponding terms in $\Delta \mathcal{L}$ arise from $t$-channel vector meson exchanges. The rationale for such an approach derives primarily from one's experience in the purely mesonic sector where, at $\mathcal{O}\left(p^{4}\right)$ in the chiral expansion, one encounters ten scale-dependent counterterms, $L_{i}^{r}(\mu)$ [53,54]. Five of these counterterms $(i=1,2,3,9,10)$ agree quite well with the predictions of vector meson exchange when the renormalization scale is chosen to be $\mu \approx m_{\rho}$. Of particular interest is the pion EM charge radius, which receives a contribution from $L_{9}^{r}(\mu)$. This counterterm contribution dominates $\left\langle r_{\pi}^{2}\right\rangle$, with the one-loop contribution giving roughly $7 \%$ of the total (for $\mu=m_{\rho}$ ). Were this situation to carry over into the arena of the nucleon's vector current form factors, one would then expect the counterterms $b^{a}$ and $c^{a}$ to be given by vector meson resonances, as shown in Fig. 2.

To explore this possibility further, one requires the couplings of $J^{P C}=1^{--}$vector mesons $V$ to spin-1/2 baryons and to electroweak vector bosons. Although it is conventional to describe the vector mesons by a vector field $V_{\mu}$, we choose instead to follow Refs. [53,54 and work with a formulation in terms of a two-index anti-symmetric tensor, $V_{\mu \nu}$. This formulation offers the advantages that (a) it is straightforward to write down a gauge- 
invariant Lagrangian for the interaction of the vector meson with electroweak vector bosons, and (b) the contributions from the diagram in Fig. 2 do not affect the normalization of the Dirac form factor at $Q^{2}=0$. In addition, one finds, as shown in Ref. [53], that the vector field formulation does not generate a vector meson contribution to the pion EM charge radius - a situation one must remedy by the introduction of an additional term at $\mathcal{O}\left(p^{4}\right)$ in the chiral Lagrangian. No such term is necessary with the tensor formulation. The primary cost involved in using the anti-symmetric tensor formulation is the presence of a four-index vector meson propagator. For the calculation of tree-level process such as given in Fig. 2, this cost is not exhorbitant. Since the details of this formulation and its relation to the vector field framework are discussed in Refs. [53,54], we refer the reader to those papers and simply give the form of the couplings and results for the nucleon form factors.

The vector meson contributions to the nucleon magnetic moment and charge radius are generated by the following two $V N N$ effective Lagrangians:

$$
\mathcal{L}_{V N N}=2 G_{T} \epsilon^{\mu \nu \alpha \beta} v_{\alpha} \bar{B}_{v} S_{\beta}^{v} B_{v} V_{\mu \nu}+\frac{G_{V}}{\Lambda_{\chi}} \bar{B}_{v} B_{v} v_{\mu} D_{\nu} V^{\mu \nu}
$$

while the gauge invariant coupling vector meson-photon coupling is given by

$$
\mathcal{L}_{V \gamma}=\frac{e F_{V} \Lambda_{\chi}}{\sqrt{2}} V_{\mu \nu} F^{\mu \nu} .
$$

A similar expression applies to the coupling of $V$ and $Z$ (the source of the baryon number current). We have omitted SU(3)-indices for simplicity. In the formalism of Refs. [53, 54], the field $V_{\mu \nu}$ has dimension one. The factors of $\Lambda_{\chi}$ have been introduced to maintain the correct dimensionality while employing dimensionless couplings. In this respect, our definition of $F_{V}$ differs from that of Refs. [53,54], where the corresponding coupling has mass dimensions. The values for the $F_{V}$ can be extracted from the rates $\Gamma\left(V \rightarrow e^{+} e^{-}\right)$. In the case of the lightest isovector vector meson, for example, one has $F_{\rho}=0.132$.

From these couplings and the amplitude associated with the diagram of Fig. 2, we find the following contributions to the Dirac and Pauli form factors:

$$
\begin{aligned}
& F_{1}^{I}\left(Q^{2}\right)=\sqrt{2} G_{V} F_{V} \frac{Q^{2}}{m_{V}^{2}-Q^{2}} \\
& F_{2}^{I}\left(Q^{2}\right)=4 \sqrt{2} G_{T} F_{V} \frac{m_{N} \Lambda_{\chi}}{m_{V}^{2}} \frac{m_{V}^{2}}{m_{V}^{2}-Q^{2}}
\end{aligned}
$$

whered $m_{V}$ is the vector meson mass. Again following Ref. [53], we have introduced the superscript $I$ to denote the results using the tensor formulation ("model $I$ " in Ref. [53]). Note that $F_{1}^{I}\left(Q^{2}=0\right)=0$, so that the vector meson resonances do not affect the nucleon charge. For purposes of comparison, we write down analagous expressions in the vector field formulation ("model $I I$ "), where one has $F_{1}^{I I}(0) \neq 0$. In this model, one must include an additional counterterm in the Lagrangian in order to preserve the nucleon's charge. Alternately, one can work with the difference $F_{1}^{I I}\left(Q^{2}\right)-F_{1}^{I I}(0)$, which is independent of the nucleon charge. Working with this difference is equivalent to making a narrow resonance approximation to a subtracted dispersion relation for $F_{1}$. Since the value of $F_{2}(0)$ is not associated with any conserved charge, no subtraction is required. The vector field formulation yields, then 


$$
\begin{aligned}
F_{1}^{I I}\left(Q^{2}\right)-F_{1}^{I I}(0) & =\frac{g_{V N N}}{f_{V}} \frac{Q^{2}}{m_{V}^{2}-Q^{2}} \\
F_{2}^{I I}\left(Q^{2}\right) & =\frac{g_{V N N} \kappa_{V}}{f_{V}} \frac{m_{V}^{2}}{m_{V}^{2}-Q^{2}}
\end{aligned}
$$

where $g_{V N N}$ and $\kappa_{V}$ give, respectively, the usual vector and tensor $V N N$ interaction strengths [55 59 and $f_{V}$ sets the scale of the $V \gamma$ transition amplitude.

From Eqs. (36-37) or (38-39), one can extract the vector meson contributions to the nucleon magnetic moment and charge radius and, thus, the corresponding contributions to the chiral coefficients $b$ and $c$ :

$$
\begin{aligned}
& b=2 \sqrt{2} G_{T} F_{V}\left(\frac{\Lambda_{\chi}}{m_{V}}\right)^{2}=\left(\frac{g_{V N N} \kappa_{V}}{f_{V}}\right)\left(\frac{\Lambda_{\chi}}{2 m_{N}}\right) \\
& c=\sqrt{2} G_{V} F_{V}\left(\frac{\Lambda_{\chi}}{m_{V}}\right)^{2}=\left(\frac{g_{V N N}}{f_{V}}\right)\left(\frac{\Lambda_{\chi}}{m_{V}}\right)^{2},
\end{aligned}
$$

where the flavor index " $a$ " has been omitted for simplicity. In the case of the nucleon's EM form factors, the expressions in Eqs. (40-41), together with the decay rates for $V \rightarrow e^{+} e^{-}$, can be used to determine the couplings $G_{V}, G_{T}$, and $F_{V}\left(\right.$ or $g_{V N N}, \kappa_{V}$, and $f_{V}$ ) [57 59]. Were one also to possess knowledge of the $F_{V}\left(\right.$ or $f_{V}$ ) associated with the strangeness matrix elements $\left\langle 0\left|\bar{s} \gamma_{\mu} s\right| V\right\rangle$, one could then use the expressions in Eqs. (40-41) to derive the counterterms for the nucleon's strangeness form factors. However, one does not at present possess such knowledge. As a fall-back strategy, one may invoke one's knowledge of the flavor content of the vector meson wavefunctions, where such knowledge exists. In doing so, it is useful to follow the spirit of Refs. [16,57,58] and write down dispersion relations for the nucleon form factors:

$$
\begin{aligned}
F_{1}^{(a)}\left(Q^{2}\right)-F_{1}^{(a)}(0) & =Q^{2} \sum_{V} \frac{a_{V}^{(a)}}{m_{V}^{2}-Q^{2}}+Q^{2} \tilde{f}_{1}^{(a)}\left(Q^{2}\right) \\
F_{2}^{(a)}\left(Q^{2}\right) & =\sum_{V} \frac{m_{V}^{2} b_{V}^{(a)}}{m_{V}^{2}-Q^{2}}+\tilde{f}_{2}^{(a)}\left(Q^{2}\right)
\end{aligned}
$$

where the superscript $(a)$ denotes the flavor channel $(T=0,1$ or strangeness), where the poles arise from vector meson exchange as in Fig. 2, and where the functions $f_{i}\left(Q^{2}\right)$ represent contributions from the multi-meson continuum. If In the works of Refs. [16,57,58, the continuum contributions were neglected in the isoscalar and strangeness channels. In the spirit of resonance saturation, we retain the leading, non-analytic loop contributions as an estimate of the continuum terms and assume that the counterterms $b^{a}$ and $c^{a}$ are dominated by the vector meson pole contributions. From Eqs. (36-43), these counterterms are easily related to the pole residues:

\footnotetext{
${ }^{4}$ Note that the continuum contribution need not enter additively; one may also include it as a multiplicative factor [59,60]. We write it additively for simplicity of illustration.
} 


$$
\begin{aligned}
b^{a} & =\left(\frac{\Lambda_{\chi}}{2 m_{N}}\right) \sum_{V} b_{V}^{(a)} \\
c^{a} & =\sum_{V} a_{V}^{(a)}\left(\frac{\Lambda_{\chi}}{m_{V}}\right)^{2} .
\end{aligned}
$$

Thus, for purposes of determining the chiral coefficients $b^{a}$ and $c^{a}$, it is just as effective to work with the residues in a pole analysis of the form factors as it is to try and determine the hadronic couplings $G_{V} G_{T}$, and $F_{V}$ (or $g_{V N N}, \kappa_{V}$, and $\left.f_{V}\right)$.

A determination of the residues was carried out by the authors of Refs. [57,58, who employed a three pole fit to the isoscalar EM form factors. The poles were identified, respectively, with the $\omega, \phi$, and one higher mass isoscalar vector meson $V^{\prime}$ (for an up-date, see Ref. 60]). The inclusion of at least two poles was needed in order to reproduce the observed dipole behavior of the isoscalar form factors. The authors found that a third pole was needed in order to obtain an acceptable $\chi^{2}$ for the fit. Subsequently, Jaffe [16] observed that since the physical $\omega$ and $\phi$ are nearly pure $u \bar{u}+d \bar{d}$ and $s \bar{s}$ states, respectively, one can relate the residues appearing in the strangeness form factor dispersion relations to those associated with the isoscalar EM form factors:

$$
\begin{aligned}
& \frac{a_{\omega}^{s}}{a_{\omega}^{T=0}}=-\sqrt{6}\left[\frac{\sin \epsilon}{\sin \left(\epsilon+\theta_{0}\right)}\right] \\
& \frac{a_{\phi}^{s}}{a_{\phi}^{T=0}}=-\sqrt{6}\left[\frac{\cos \epsilon}{\cos \left(\epsilon+\theta_{0}\right)}\right],
\end{aligned}
$$

where $\epsilon$ is the mixing angle between the pure $u \bar{u}+d \bar{d}$ and pure $s \bar{s}$ states and $\theta_{0}$ is the "magic" octet-singlet mixing angle giving rise to these pure states. Analogous formulae apply for the residues appearing in the expressions for $F_{2}$. From Eqs. (44-47), one may now determine the $\omega$ and $\phi$ contributions to the constants $b^{s}$ and $c^{s}$.

A determination of the remaining residues $a_{V^{\prime}}^{s}$ and $b_{V^{\prime}}^{s}$ is more problematic. One does not possess sufficient knowledge of the $V^{\prime}$ flavor content to derive a simple relation between the strangeness and isoscalar EM residues. One must therefore employ alternative strategies. Jaffe arrived at values for the $a_{V^{\prime}}^{s}$ and $b_{V^{\prime}}^{s}$ by imposing conditions on the asymptotic behavior of the form factors $\left(Q^{2} \rightarrow \infty\right)$. Using a three pole fit, with all masses and two residues fixed, one is only able to require that $F_{1}$ vanish as $1 / Q^{2}$ and $F_{2}$ as $1 / Q^{4}$. These asymptotic conditions are more gentle than one would expect based on the most naïve quark counting rules. Consistency with the latter would require the inclusion of more poles with unknown masses and residues than used in the fits of Refs. [16, 57, 58]. Since the adequacy of these quark counting rules for strangeness form factors is itself not clear, and since one's predictions for the nucleon's strangeness radius and magnetic moment within this framework are nontrivially dependent on one's assumptions about asymptopia, this approach to treating the $V^{\prime}$ contribution is ambiguous at best.

Another alternative is to note that in the fits of Ref. [58], the $V^{\prime}$ contributes very little to the isoscalar mean square radius and anomalous magnetic moment (less than $10 \%$ in the fits with the best $\chi^{2}$ ). Indeed, the primary benefit of including the $V^{\prime}$ was to obtain acceptable $\chi^{2}$ over the full range of $Q^{2}$ used in the fit; it's impact on the value of the form factors and their slopes at the origin is minimal. The latter result is not surprising, since 
the $V^{\prime}$ contribution to the low- $\left|Q^{2}\right|$ behavior of the form factors is suppressed by powers of $\left(m_{\omega, \phi} / m_{V^{\prime}}\right)^{2}$ relative to the $\omega$ and $\phi$ contributions. It would seem reasonable, then, to neglect the $V^{\prime}$ when seeking to determine the leading, non-trivial $Q^{2}$ behavior of the strangeness form factors. This strategy is the one we adopt here. The uncertainty associated with neglecting the $V^{\prime}$ pole is certainly no greater than the ambiguity one encounters when using large $Q^{2}$ conditions to determine low-momentum constants. Moreover, in the present study, we seek to make statements only about the strangeness radius and magnetic moment, and not about the full $Q^{2}$-dependence of the strangeness form factors. Hence, including additional poles beyond the $\omega$ and $\phi$ is not a strong necessity. In principle, the $V^{\prime}$ or even higher mass isoscalar $1^{--}$resonances could generate large contributions to $b^{s}$ and $c^{s}$. Such result would be surprising, based on the situation in the isoscalar channel. Nevertheless, the latter possibility cannot be ruled out on general grounds. Indeed, one's lack of knowledge of the contributions from the $V^{\prime}$ and beyond constitutes one of the weak points in the resonance saturation model. To be on the conservative side, we choose to omit contributions about which we have no knowledge.

In Table I, we quote results for the nucleon's Dirac strangeness radius and magnetic moment assuming the $\omega$ and $\phi$ residues saturate the constants $b^{s}$ and $c^{s}$. We obtain these constants using the Jaffe relations in Eq. (30), the results from fit 8.2 of Ref. [58] (which gives the best $\chi^{2}$ ), and Eqs. (44-45). In viewing these results, a few additional caveats should be kept in mind. First, the one-loop contributions to the isoscalar and strangeness radius and magnetic moment quoted above represent a subset of a larger class of continuum contributions to the moments. In the language of Eqs. (42-43), these loop calculations give an estimate of the two kaon intermediate state contributions to the functions $\tilde{f}\left(Q^{2}\right)$. Contributions from the $3 \pi, 5 \pi, K K \pi, \ldots$ states have not been included.

Second, the resonance saturation model is only partially successful in the case of the nucleon's EM moments, in contrast to the situation with the pion form factor. To illustrate, we consider the EM Dirac charge radii. In the case of the isovector radius, the loop contribution is signficantly larger than the experimental value: $\rho_{L O O P}^{T=1} / \rho_{E X P T}^{T=1} \approx 1.5\left(\right.$ taking $\left.\mu \approx m_{\rho}\right)$. [ One therefore requires a contribution from $c^{T=1}$ which cancels about $40 \%$ of the loop contribution. The $\rho$ meson contribution to $c^{T=1}$, computed using the values of $f_{V}$ taken from $e^{+} e^{-}$data and $g_{V N N}$ determined from fits to $N N$ scattering amplitudes [56] has the wrong sign to bring about this cancellation. In fact, a careful analysis of the isovector spectral function for the Dirac form factor, which contains information about both the continuum and $\rho$ resonance contributions, can be used to extract a value for $g_{V N N}$ consistent with the value used in $N N$ scattering studies [59]. Such an analysis includes pion rescattering corrections which reduce the two pion continuum contribution and allow for a larger $\rho$ pole term. In the case of the isoscalar Dirac radius, on the other hand, the kaon loop contribution to the isoscalar Dirac radius is about $15 \%$ of the experimental value. To the extent that the

\footnotetext{
${ }^{5}$ In the work of Ref. 61, only $\pi$ loops were considered and the result for $\rho_{L O O P}^{T=1}$ is closer to the experimental value. Our result also includes the $K$-loop contribution. Although the calculation of Ref. [61] was carried out without using the heavy baryon formalism, the result agrees with the chiral log of the heavy baryon calculation. The results for the magnetic moment differ, however.
} 
multi-pion continuum and kaon rescattering contributions are neglegible, $\rho_{D}^{T=0}$ is therefore resonance dominated.

Third, one must note the presence of an issue of consistency between the resonance saturation model employed here and the ways in which the constants $b^{s}$ and $c^{s}$ have been extracted. In the analysis of Refs. [57,58], no continuum contributions were included in the fit to the isoscalar form factors. Such an approximation may be valid in the case of the isoscalar Dirac radius, for which the loop contributions represent a reasonably small fraction of the total. One might expect, then, that a fit which included the $2 K$ continuum contributions would not yield residues $a_{\omega, \phi}^{T=0}$ differing appreciably from the values of Refs. [57].58]. Stated differently, not only is the constant $c^{T=0}$ dominated by resonance, but apparently so is the entire isoscalar Dirac radius. Conversely, the $\mathcal{O}\left(\sqrt{m_{s}}\right)$ kaon loop contribution to the isoscalar anomalous magnetic moment is large: $\kappa_{L O O P}^{T=0} / \kappa_{E X P T}^{T=0} \approx 20$. Chiral perturbation theory therefore requires a large constant $b^{T=0}$ to cancel most of this loop contribution. Unfortunately, the most reliable information one has on the resonance contributions to the isoscalar magnetic form factor is derived from the fits of Ref. [58, which included no continuum. The residues $b_{\omega, \phi}^{T=0}$ obtained from these fits are small (on the order of $\kappa_{E X P T}^{T=0}$ ) and, therefore, cannot cancel the large kaon loop contribution. Presumably, a reanalysis of the isoscalar magnetic form factor which included the kaon continuum at one-loop order would yield larger values for the residues. We would conclude from these observations that, of the resonance saturation predictions quoted in Table I, the value for the Dirac radius is the more credible.

Kaon cloud dominance. A second possibility is to relax the requirement that one undertake a consistent chiral expansion and use kaon loops alone to make a prediction. The rationale for this approach has a two-fold basis. The first follows from a geometric interpretation of the nucleon charge radius, wherein it characterizes a spatial asymmetry in the charge distribution. In this picture, a spatial polarization of the strange sea arises from fluctuations of the nucleon into a kaon and strange baryon. The kaon, having about half the mass of the lightest strange baryons lives on average further from the nucleon center of mass than the strange baryon. One would expect, then, to obtain a negative value for $\left\langle r_{s}^{2}\right\rangle$ (positive value for $\rho^{s}$ ), since the kaon carries the $\bar{s}$. Implicit in this picture is an assumption that $s \bar{s}$ pair creation by the neutral gauge boson probe, which also contributes to the Dirac or electric form factors and which appears partially in the guise of resonance contributions, is negligible compared to the mechanism of $s \bar{s}$ spatial polarization. The kaon cloud dominance approach also assumes that the multi-pion contribution is negligible when compared to that of the kaon cloud, ostensibly because the pion contains no valence $s$ or $\bar{s}$ quarks.

The second motivation draws on the result of a pion loop calculation of the nucleon's EM form factors first carried out by Bethe and DeHoffman [62]. This calculation was performed using the equivalent of the linear $\sigma$-model. At the time they were reported, the results were in surprising agreement with the experimental values for the nucleon's charge radii and magnetic moments, despite the large value of the $\pi N$ coupling which enters this perturbative calculation. The lore which developed in the aftermath of this calculation is that the pion cloud dominates the nucleon's isovector EM moments and that a one-loop calculation sufficiently incorporates the physics of the pion cloud. Were this situation to persist in the strangeness sector, one would expect that the kaon cloud gives the dominant 
contribution to the $\rho^{s}$ and $\mu^{s}$ and that a one loop calculation would suffice to give their correct magnitude and sign.

A variety of one-loop calculations have been performed assuming that the kaon cloud dominates the strange form factors. For example, the authors of Ref. [17 computed $\rho_{s}$ and $\mu_{s}$ within the context of the $\mathrm{SU}(3)$ linear $\sigma$-model. Within this framework, the leading strangeness moments are U.V. finite. Nevertheless, the calculation was performed by including hadronic form factors at the $K N \Lambda$ vertices, drawing on results of fits to baryonbaryon scattering in the one meson exchange approximation which find better agreement with data if hadronic form factors are included. The authors of Refs. [19,20 extended this approach to compute both the leading moments as well as the non-leading $Q^{2}$-dependence of the strangeness form factors using a hybrid kaon-loop/vector-meson pole model. Although the hybrid model goes beyond a simple one-loop approximation, it nevertheless represents a type of kaon cloud model inasmuch as non-resonant multi-pion contributions are omitted. Another variation of this general approach is a study performed using the cloudy bag model $(\mathrm{CBM})$ and the "cloudy" constituent quark model (CCQM) 18. The CBM represents a kind of marriage of the MIT bag model with spontaneously broken chiral symmetry. The strength of the meson-baryon vertices is determined by the meson-quark coupling and the quark's bag model wavefunction. The CCQM is similar in spirit, though in this case the nonrelativistic constituent quarks are confined with a harmonic oscillator potential. In effect, the CBM and CCQM calculations represent kaon loop calculations in which the $N \Lambda K$ and $N \Sigma K$ form factors are determined by the dynamics of the particular models. More recently, Geiger and Isgur have extended the kaon cloud idea to include one loop contributions from all known strange mesons and baryons using the non-relativistic quark model to obtain a nucleon/strange hadron vertex function 25].

In all cases, these models include contributions which are both non-analytic and analytic in the $s$-quark mass, effectively modelling contributions from the relevant higher-dimension operators appearing in an effective Lagrangian. Moreover, in each instance the loop integration was cut-off at some momentum scale by including form factors at the hadronic vertices. In both respects, a consistent chiral expansion is lost. In principle, higher-order loop contributions could yield terms of the same chiral order as some of the analytic terms retained from the one-loop amplitudes. Similarly, the use of hadronic form factors with a cut-off parameter breaks the consistency of the expansion because a new scale is introduced (e.g., the 1/hadron size) and/or because the form factor itself contributes like an infinite tower of higher-dimension operators.

One might argue that since $m_{K} / \Lambda_{\chi}$ is not small, the chiral expansion is not all that useful in the case of strange quarks and that models which are inconsistent with this expansion may yet be credible [63,64]. We wish to illustrate, nonetheless, that the approach of kaon cloud dominance still presents a host of uncertainties. To do so, we repeat the calculation of Ref. [17] in the framework of the non-linear $\mathrm{SU}(3) \sigma$-model, corresponding to the meson-baryon Lagrangian of Eq. (10). In this case, the strangeness radius is U.V. divergent, unless one includes form factors at the hadronic vertices. A simple choice, and one which renders the loop calculation most tractable, is the monopole form:

$$
F\left(k^{2}\right)=\frac{m_{K}^{2}-\Lambda^{2}}{k^{2}-\Lambda^{2}}
$$


where $k$ is the momentum of the kaon appearing at the $K N \Lambda$ vertex and $\Lambda$ is a momentum cut-off. The monopole form above was employed in the Bonn potential fits to baryon-baryon scattering, and value of the cut-off $\Lambda \sim 1.2-1.4 \mathrm{GeV}$ was obtained [56]. Various kaon cloud models differ, in part, through the choice of form for $F\left(k^{2}\right)$ and the value of the cut-off parameter.

The inclusion of a hadronic form factor necessitates the introduction of additional, "seagull" graphs in order to maintain the gauge invariance of the calculation (Fig. 1c,d). Without these new graphs, the loop calculation with hadronic form factors does not satisfy the vector current Ward-Takahashi identity. It was shown in Refs. [17,[19] that use of the minimal subsitution $k_{\mu} \rightarrow k_{\mu}+i \hat{Q} Z_{\mu}$ in $F\left(k^{2}\right)$ generates a set of seagull vertices whose loop graphs restore agreement of the calculation with the $\mathrm{W}$-T identity. It is straightforward to show that for a meson-nucleon vertex of the form

$$
\mp i F\left(k^{2}\right) k^{\lambda} \tilde{\Pi} \quad \bar{u} \gamma_{\lambda} \gamma_{5} u
$$

the corresponding seagull vertex is

$$
\left\{ \pm i Z_{\mu}\left(Q^{\mu} \pm 2 k^{\mu}\right) \frac{\left[F\left((Q \pm k)^{2}\right)-F\left(k^{2}\right)\right]}{\left[(Q \pm k)^{2}-k^{2}\right]} k^{\lambda}[\hat{Q}, \tilde{\Pi}]+i Z^{\lambda} F\left((Q \pm k)^{2}\right)[\hat{Q}, \tilde{\Pi}]\right\} \quad \bar{u} \gamma_{\lambda} \gamma_{5} u
$$

where $\tilde{\Pi}$ is the pseudoscalar octet matrix defined in Section II, $Q_{\mu}$ is the momentum of the source $Z$ (for EM or baryon number current), $\hat{Q}$ is the corresponding charge, and where the upper (lower) sign corresponds to in incoming (outgoing) meson.

In Section IV and Table I we give the results of the kaon loop calculation using the nonlinear $\mathrm{SU}(3) \sigma$-model and hadronic form factors (as in Eq. (47)) as a function of the cut-off $\Lambda$. We compare these results with those of other kaon cloud models in order to estimate the range in predictions which arises under the rubric of kaon cloud dominance. Indeed, the existence of such a range reflects the ambiguities associated with this general approach. First, as mentioned previously, one has already abandoned a consistent chiral expansion. Consequently, one has no rigorous justification for retaining only one-loop contributions. Second, the choice of hadronic form factor is not unique. In the CBM, for example, the form of the effective $F\left(k^{2}\right)$ is approximately Gaussian rather than monopole as used here. Moreover, the scale of the momentum cut-off is set by the inverse bag radius, which is on the order of a few hundred $\mathrm{MeV}$ [18]. The CBM constitutes a chiral model with a different underlying physical picture than the non-linear $\sigma$-model, and its parameters can be tuned to produce agreement with at least some of the nucleon's EM form factors. One has no strong phenomenological reason, then, to choose one model - corresponding to one form for $F\left(k^{2}\right)$ - over another; only a model preference. Third, the prescription for maintaining gauge invariance is also not unique. The one shown above is a minimal procedure. One may include additional seagull contributions which are purely transverse and, therefore, do not affect the $\mathrm{W}-\mathrm{T}$ identity. The presence of these additional terms may, nevertheless, affect one's results for the form factors. Finally, this approach omits resonance contributions altogether. This omission by itself ought to raise concern. Indeed, it has long been known, from dispersion theoretic studies, that a significant part of the nucleon's isovector EM form factors contain important resonance contributions [59]. 
Constituent quarks. The final model approach we consider entails treating the nucleon's strangeness matrix elements as arising from the strangeness "content" of constituent $U$ - and $D$-quarks. The motivation for this approach derives from a picture of the constituent quark as a current quark of QCD surrounded by a sea of gluons and $q \bar{q}$ pairs. It follows that the nucleon's strangeness radius and magnetic moment arise from the corresponding quantities for the consituent $U$ - and $D$-quarks 65. The procedure one follows within this framework is essentially the quark model analog of the one-body approximation made in computing nuclear current matrix elements [24]. Specifically, one derives an operator associated with the individual constituents (quarks, nucleons) and computes a matrix element of that operator using the appropriate bound state (hadron, nucleus) wavefunction. Chiral symmetry is invoked in deriving the constituent quark strangeness current operators. Such a calculation of $\rho_{S}^{s}$ was performed by the authors of Ref. [19], using the Nambu/Jona-Lasinio (NJL) model [66] to compute the constituent quark strangeness radii.

An alternative is to adopt a chiral quark model framework, wherein the constituent quark strangeness currents arise from fluctuations of the $U$ - and $D$-quarks into a kaon plus a constituent $S$-quark. The contributions from the individual $U$ - and $D$-quarks are added to give the total nucleon strangeness matrix element using a quark model spin-space-flavor wavefunction, as illustrated schematically in Fig. 3. The strength of the kaon-constituent quark interaction is governed by the parameter $g_{A}$ appearing in the chiral Lagrangian of Eq. (7). This parameter can be determined by using the constituent chiral quark model to compute the nucleon's axial vector current. Since $g_{A}$ enters the strangeness matrix element of the nucleon at one-loop order, one need only determine it at tree level (see below).

It is worth noting that the chiral quark model does not suffer from the same lack of convergence which plagues the naïve baryon chiral Lagrangian due to the size of $m_{B}$. Since the constituent quark mass is considerably smaller than $\Lambda_{\chi}$, one has reason to believe that higher-order corrections to the leading order Lagrangian in Eq. (7), as well as higher-order loop effects, will be suppressed. On the other hand, the ambiguity associated with the coefficients $b^{s}$ and $c^{s}$ remains. In the case of chiral quarks, one may still write down corrections to $\mathcal{L}_{Q}$ associated with the magnetic moment and Dirac charge radius of a constituent quark that are, respectively, of lower order and the same order in $1 / \Lambda_{\chi}$ as the corresponding contributions from loops:

$$
\Delta \mathcal{L}=\frac{b_{q}^{a}}{2 \Lambda_{\chi}} \bar{\psi} \sigma_{\mu \nu} \hat{Q} \psi F^{\mu \nu}-\frac{c_{q}^{a}}{\Lambda_{\chi}^{2}} \bar{\psi} \gamma_{\mu} \hat{Q} \psi \partial_{\nu} F^{\mu \nu},
$$

where $\hat{Q}$ is the appropriate charge (EM or baryon number), $F^{\mu \nu}$ is the field strength associated with the corresponding source, and the " $a$ " superscript denotes the flavor channel [67].

As in the case of the baryon chiral Lagrangian, the coefficients $b_{q}^{0}$ and $c_{q}^{0}$ in the $\mathrm{SU}(3)$ singlet channel cannot be determined from known moments. Consequently, one must invoke additional model assumptions in order to make chiral quark model predictions for the

\footnotetext{
${ }^{6}$ The CCQM calculations of Ref. [18] omit contributions from the strangeness content of the constituent quarks. Only the kaon cloud around the entire bag of quarks is considered.
} 
nucleon's strangeness matrix elements. In the present study, we adopt the following strategy. First, we simply omit the contributions from the $b_{q}^{a}$ and $c_{q}^{a}$ and take the constituentquark/kaon one-loop contribution as an indication of the scale of the constituent quark strangeness radius and magnetic moment. Although this assumption, which represents our model ansatz, may appear to be a drastic approximation, it is no more questionable than would be any attempts to make model predictions for the singlet coefficients $b_{q}^{0}$ and $c_{q}^{0}$. Second, we cut the loops off at $\Lambda_{\chi}$, effectively restricting the virtual Goldstone bosons to have momenta less than the scale of chiral symmetry breaking. An alternative would be to use dimensional regularization and subtract terms proportional to $\mathcal{C}_{\infty}$ (equivalent to $\overline{\mathrm{MS}}$ renormalization). Since we are interested only in obtaining the scale of the constituent $U$ and $D$-quark strangeness current and not in making airtight predictions, either approach would suffice. In order to cut the loops off in a gauge invariant manner, we employ form factors at the quark-kaon vertices introducing the appropriate seagull graphs as necessary to preserve the W-T identities. For simplicity, we use the monople form of Eq. (47), taking the cut-off parameter $\Lambda \sim \Lambda_{\chi}$. In effect, we repeat the non-linear $\sigma$-model calculation discussed above for constituent quarks rather than nucleons.

The results of the loop calculation generate effective, constituent quark strangeness current operators

$$
\left.\left\langle Q\left|\bar{s} \gamma_{\mu} s\right| Q\right\rangle\right|_{L O O P} \rightarrow \hat{J}_{\mu}^{S T R A N G E}=\hat{\bar{\psi}}\left[F_{1 Q}^{(s)} \gamma_{\mu}+\frac{i F_{2 Q}^{(2)}}{2 m_{Q}} \sigma_{\mu \nu} Q^{\nu}\right] \hat{\psi}
$$

where $Q$ denotes a constituent quark and $\hat{\psi}$ is a constituent quark field operator. Nucleon matrix elements of $\hat{J}_{\mu}^{S T R A N G E}$ may be computed using quark model wavefunctions. We choose to employ wavefunctions in the light-front formalism, since this framework allows one to use the on-shell constituent quark current (the form in Eq. (51) and allows one to perform boosts along the direction of momentum transfer as needed to properly account for the nucleon's center of mass motion. Although we are concerned only with the leading, nontrivial $Q^{2}$-dependence of the strangeness form factors, it is worth noting that the light-front quark model has successfully reproduced the the nucleon's EM form factors over a significant range in momentum-transfer [68 70]. We also follow the authors of Ref. [70], who take a tree-level value for the meson-quark coupling $g_{A}=1.0$ and an oscillator parameter $\gamma=1.93$ $\mathrm{fm}^{-1}$ and reproduce the nucleon's isovector axial charge to within $5 \%$.

As in the case of the other model approaches discussed here, one should note the shortcomings of the chiral quark model. Perhaps the primary difficulty is a conceptual one involving double counting. As noted in Ref. [47], the chiral quark effective theory contains both the pseudoscalar $Q \bar{Q}$ bound states as well as the octet of light pseudoscalar Goldstone bosons. To the extent that the latter are also $Q \bar{Q}$ bound states, the theory contains the same set of states in two different guises. The authors of Ref. [47] argue, based on a simple Goldstone boson- $Q \bar{Q}$ mixing diagram, that the mass of the bound state must be either somewhat greater than $\Lambda_{\chi}$, in which case it lies outside the realm of the low-energy effective theory, or infinity, in which case it is unphysical. One would conclude that the Goldstone boson octet is distinct from the lightest $Q \bar{Q}$ states of the theory. A study of meson spectroscopy, however, suggests otherwise. Indeed, the pattern of mass splittings in the $B B^{*}$, $D D^{*}, K K^{*}$ and $\pi \rho$ systems is remarkably consistent with the mass splittings in conventional 
quarkonia between the lightest ${ }^{3} S_{1}$ and ${ }^{1} S_{0} Q \bar{Q}$ states [71. This pattern strongly suggests that the Goldstone bosons are the lightest $Q \bar{Q}$ bounds states of the effective theory [72], in conflict with the conclusions of Ref. [47]. There do exist methods for constructing a chiral quark effective theory which includes mesons as specific degrees of freedom while avoiding the double counting problem (see, e.g. Ref. [73]). However, performing a calculation at this level of sophistication lies beyond the scope of the present study. Our goal is simply to illustrate how predictions for $\rho^{s}$ and $\mu^{s}$ compare between models where chiral symmetry is invoked at a microscopic level and those in which it is included at the purely hadronic level.

\section{RESULTS AND DISCUSSION}

In this section, we give predictions for the nucleon's strangeness radius and magnetic moment using the three chiral model approaches discussed above. These results are summarized in Table I, where we also give predictions from four previously reported approaches sharing some elements in common with those discussed here. For illustrative purposes, we also display in Fig. 4 the dependence of $\rho_{s}$ and $\mu_{s}$ on the hadronic form factor cut-off parameter and pseudoscalar meson mass entering the non-linear $\sigma$-model calculation.

\begin{tabular}{|c|c|c|c|}
\hline \multicolumn{4}{|c|}{ TABLE I } \\
\hline Model & $\rho_{D}^{s}$ & $\mu^{s}$ & $\rho_{S}^{s}$ \\
\hline Resonance Sat. ${ }^{(a)}$ & $-3.62(1.52)$ & $1.85(2.2)$ & $-5.47(-0.68)$ \\
\hline $\mathrm{NL} \Sigma \mathrm{M} / \mathrm{FF}^{(b)}$ & 0.11 & -0.25 & 0.36 \\
\hline Chiral Quarks $^{(c)}$ & 0.53 & -0.09 & 0.62 \\
\hline Poles $^{(d)}$ & $-2.43 \pm 1.0$ & $-0.31 \pm 0.009$ & $-2.12 \pm 1.0$ \\
\hline $\mathrm{L} \Sigma \mathrm{M} / \mathrm{FF}^{(e)}$ & 0.1 & $-(0.31-0.40)$ & $0.41-0.49$ \\
\hline $\operatorname{Hybrid}^{(f)}$ & 0.37 & $-(0.24-0.32)$ & $0.61-0.68$ \\
\hline $\mathrm{CBM}^{(g)}$ & 0.15 & -0.09 & 0.24 \\
\hline
\end{tabular}

Table I. Theoretical predictions for nucleon strange quark vector current form factors. Columns two and three give dimensionless mean square Dirac strangeness radius and strangeness anomalous magnetic moment, respectively. The fourth column gives the Sachs strangeness radius: $\rho_{S}^{s}=\rho_{D}^{s}-\mu^{s}$. To convert to $\left\langle r_{s}^{2}\right\rangle$, multiply $\rho^{s}$ by $-0.066 \mathrm{fm}^{2}$. First three lines give predictions of chiral models discussed in this work: (a) heavy baryon $\mathrm{CHPT} /$ resonance saturation employing $\omega$ and $\phi$ residues of Fit. 8.2 of Ref. [58]; numbers in parentheses give loop contribution for $\mu=m_{\rho}$; (b) non-linear $\sigma$-model with hadronic form factors using cutoff mass $\Lambda=1.2 \mathrm{GeV}$; (c) chiral quark model with cutoff mass $\Lambda=1.0$ $\mathrm{GeV}$, oscillator parameter $\gamma=1.93 \mathrm{fm}^{-1}$, and $m_{U}=m_{D}=0.33 \mathrm{GeV}$. Last four lines give 
previously reported predictions: (d) three pole model of Ref. [16]; (e) linear $\sigma$-model of Ref. [17; (f) hybrid pole/loop model of Ref. [20]; (g) cloudy bag model of Ref. [18].

When viewed from the most "impressionistic" perspective, the results in Table I illustrate the wide spread in predictions one encounters among approaches relying on chiral symmetry. Indeed, the strangeness radius and magnetic moment can vary by an order of magnitude and by sign. One ought to conclude that chiral symmetry by itself is not a terribly restrictive input principle when it comes to predicting nucleon strangeness. The reason is essentially that the quantity one wishes to predict is the very quantity one needs in order to make a prediction - the $\mathrm{SU}(3)$-singlet vector current moments. In the absence of experimental information on the latter, the range in one's predictions can be as wide as the breadth of one's space of chiral models. From the standpoint of hadron structure theory, this situation is not very satisfying, since one would like to possess a reliable effective-theory framework for interpreting the up-coming measurements of the low-energy properities of the $s \bar{s}$ sea.

Nevertheless, one may still ask whether there exist reasons to give greater credibility to one approach over the others. In this respect, we admit to some bias in favor of the resonance saturation prediction for the Dirac strangeness radius - if only because it appears to suffer from fewer ambiguities than the other approaches. The reasons for this bias can be summarized as follows:

(a) Detailed analyses of the isovector charge radius imply that it is dominated by the lowest continuum state (two pions) and the lightest isovector $1^{--}$resonance. Although a simple one pion loop plus $\rho$ resonance calculation over-predicts the isovector charge radius by a factor of two [50], the sign and order of magnitude are given correctly. The over-prediction appears to result from the omission of non-resonant pion rescattering corrections [52].

(b) One would expect a similar situation to persist in the isoscalar and strangeness channels, where the lightest continuum states are the $3 \pi, 5 \pi, 7 \pi$, and $2 K$ states and the lightest isoscalar $1^{--}$vector mesons are the $\omega$ and $\phi$. Some thought about chiral counting suggests that the multi-pion contributions ought to be suppressed relative to the $2 K$ contribution. To the extent that this suppression holds and that the total, non-resonant $2 K$ contribution to the isoscalar charge radius is at least as small as given the result of the one-loop heavy baryon calculation, the isoscalar charge radius would then be dominated by the lightest isoscalar vector mesons, rendering the fit of Ref. [58] quite valid. The results of this fit indicate that the $\omega$ and $\phi$ residues dominate the isoscalar Dirac radius; the contribution from higher mass vector mesons is negligible. Thus, the isoscalar constants $b^{T=0}$ and $c^{T=0}$ should be given quite reliably by the $\omega$ and $\phi$ contributions.

(c) Knowledge of the $\omega$ and $\phi$ flavor content allows one to translate the $\omega$ and $\phi$ contributions to the isoscalar constants into the corresponding contributions to the strangeness constants, $b^{s}$ and $c^{s}$. If the non-resonant multi-pion contributions to the strangeness radius are suppressed with respect to the $2 K$ contribution, if the kaon loop contribution (Eq. (31)) accurately reflects the scale of the two kaon continuum, and if there are no important vector meson effects beyond those of the $\omega$ and $\phi$, then $\rho_{D}^{s}$ ought to be given accurately by resonance saturation model.

One should note that this line of argument avoids the problematic use of assumptions about the strangeness form factors' large $Q^{2}$ behavior while incorporating the consistency of the heavy baryon chiral expansion. 
The logic of this reasoning could break down in a number of ways. Were a careful dispersion analysis of the isoscalar form factors to reveal large multi-pion or kaon rescattering contributions, the pole fit of Ref. [58] would need to be re-done, presumably leading to modified values for the vector meson residues. Moreover, such a situation would imply the presence of important non-resonant multi-pion and kaon rescattering contributions to the strangeness form factors as well. In addition, one cannot a priori rule out important contributions from higher mass intermediate states, even though there is little evidence for such contributions in the isovector and isoscalar charge radii.

What about the strangeness magnetic moment? In this case, the resonance saturation prediction is highly questionable. The reason is that the heavy baryon calculation gives a large loop contribution to $\kappa^{T=0}$. Were this result to accurately reflect a large non-resonant kaon continuum contribution, the pole fit of Ref. [58] would be invalid and derived constants $b^{T=0}$ and $b^{s}$ un-reliable. On the other hand, since $m_{K} / \Lambda_{\chi}$ is not small, it would not be surprising to find important kaon rescattering corrections which could cancel the leading term. In short, the magnitude and sign of the continuum contributions to $\kappa^{T=0}$ is uncertain. This uncertainty in turn raises doubts about the resonance saturation prediction for $\mu^{s}$. One should also keep in mind that the questionability of this prediction for $\mu^{s}$ implies that the prediction for the Sachs radius is also questionable, since $\rho_{S}^{s}=\rho_{D}^{s}-\mu^{s}$.

Other model uncertainties. The remaining model predictions listed in Table I carry as large, if not a greater degree of ambiguity than the resonance saturation prediction for $\mu^{s}$.

1.Kaon cloud dominance. Kaon cloud dominance models can be challenged on at least three grounds: (a) They sacrifice consistent chiral counting in order to make predictions. This sacrifice comes about through the introduction of form factors at the hadronic vertices and through the retention of terms in the one-loop amplitudes which are both analytic and nonanalytic in the strange quark mass. Terms of the former class are indistinguishable from contributions arising from higher-dimension operators in the chiral Lagrangian. Moreover, higher-order loop graphs may yield analytic terms of the same chiral order as some of the analytic terms retained from the one-loop graphs. A consistent chiral approach would require the inclusion of all analytic contributions of a given order in $1 / \Lambda_{\chi}$. (b) They do not include resonant $t$-channel kaon rescattering or multi-pion contributions (both resonant and nonresonant). (c) As noted previously, one encounters ambiguities associated with one's choice of form for the hadronic form factors, the size of the cut-off parameter, and the transverse part of the covariantizing seagull graphs.

It is instructive to try and quantify the uncertainty associated with these ambiguities. This effort is most easily accomplished for those of type (c) by considering the $\Lambda$-dependence of the linear and non-linear $\sigma$-model predictions and by comparing these predictions with those of the CBM and CCQM calculations. Turning first to the issue of the cut-off dependence, one may argue about which value of $\Lambda$ to use. The results quoted in Table I and Ref. [17 for the linear $\sigma$-model were obtained using the Bonn value, $\Lambda \sim \Lambda_{B O N N} \sim 1.2 \mathrm{GeV}$. According to the fits of Ref. [56], taking $\Lambda \sim \Lambda_{B O N N}$ optimizes agreement with baryon-baryon scattering data in the one meson-exchange approximation. For this choice of $\Lambda$, however, the corresponding pion loop contributions to the EM moments are in serious disagreement with the experimental values. In fact, there exists no value of $\Lambda$ which produces agreement between experiment and the linear $\sigma$-model values for the EM moments. The best choice 
occurs for $\Lambda \approx 5 \mathrm{GeV}$. In this case, experiment and the linear $\sigma$-model agree for $\kappa^{T=1}$ while the prediction for $\rho^{T=1}$ is $60 \%$ of the experimental value. Changing $\Lambda$ from $\Lambda_{B O N N}$ to $\Lambda \approx 5$ $\mathrm{GeV}$ doubles the prediction for $\mu^{s}$ and reduces the prediction for $\rho_{D}^{s}$ by $25 \%$.

The choice of $\Lambda$ in the case of the non-linear $\sigma$-model is equally debatable, as a study of the pion-loop contribution to the isovector magnetic moment illustrates. We find no value of the cut-off which reproduces the experimental value. Choosing $\Lambda \sim \Lambda_{\chi} \sim \Lambda_{B O N N}$ yields, for example, $\kappa_{L O O P}^{T=1} / \kappa_{E X P T}^{T=1} \approx 25 \%$ ( the corresponding ratio for the isovector Dirac radius is $\rho_{L O O P}^{T=1} / \rho_{E X P T}^{T=1} \approx 27 \%$ ). Taking the limit $\Lambda \rightarrow \infty$ gives $\kappa_{L O O P}^{T=1} / \kappa_{E X P T}^{T=1} \approx 54 \%$ (the isovector Dirac radius diverges in this limit). One might argue, then, that choosing any value of the cut-off in the range $\Lambda_{\chi} \leq \Lambda \leq \infty$ would be equally justified - at least for the magnetic moments which are U.V. finite. As the cutoff is varied over this range, $\mu^{s}$ varies from the value quoted in Table I to $\mu^{s}=-1.31$. This situation appears to persist in the case of the CBM and CCQM models as well. The authors of Ref. [18] originally found values for the cutoff which optimized agreement with all the nucleon's EM moments. However, a subsequent inclusion of covariantizing seagull graphs changed the magnetic moment predictions by $50 \%$ so that an optimum cutoff no longer exists. Consequently, the corresponding predictions for the strangeness moments contains an uncertainty associated with the value of the cut-off.

A comparison between different kaon cloud models reveals a similar degree of ambiguity. We consider first the linear and non-linear $\sigma$-models. When one of the baryons is off shell, as is the case in a loop calculation, the structure of the meson-baryon vertices differ in the two models, even though the same monopole form factor was used in both calculations. When one takes $\Lambda \sim \Lambda_{\chi} \sim \Lambda_{B O N N}$, the two models give nearly identical predictions for $\rho_{D}^{s}$. One might not be surprised by this result, since in both cases the radius contains a chiral log. At least in the chiral limit, this infrared singularity dominates over contributions analytic in $m_{K}$, and it is essentially terms of the latter type which would be responsible for any differences in the two predictions. For $\Lambda \rightarrow \infty$, on the other hand, $\rho_{D}^{s}$ diverges in the non-linear $\sigma$-model but only doubles in value in the linear $\sigma$-model. In the case of the strangeness magnetic moments, which contain no infrared or ultraviolate singularities, the model predictions differ by a factor of about 1.5 for $\Lambda \sim \Lambda_{\chi}$ 马 but come into closer agreement for $\Lambda \rightarrow \infty$. Comparing the CBM and $\sigma$-model $\left(\Lambda=\Lambda_{B O N N}\right)$ predictions, one finds CBM gives a $50 \%$ larger Dirac radius but a value for $\mu^{s}$ that is a factor of three or four smaller than the linear $\sigma$-model prediction.

These comparisons are not definitive. Nevertheless, they suggest a scale for uncertainty in the kaon cloud dominance predictions that amounts to about a factor of five or more times the smallest values for $\left|\rho_{D}^{s}\right|$ and $\left|\mu^{s}\right|$. This rather large model-spread reflects two of the weaknesses of the kaon cloud dominance approach: (a) the lack of a systematic expansion

\footnotetext{
${ }^{7}$ One would not expect the pion loop graphs to produce agreement with the isoscalar moments. As the heavy baryon calculation illustrates, the leading contribution arises from the diagrams where the current is inserted in the meson line. In the case of the pion loops, these diagrams only contribute to the isovector moments.
}

${ }^{8}$ The lower value for $\left|\mu^{s}\right|$ in the case of the linear $\sigma$-model corresponds to $\Lambda=1.2 \mathrm{GeV}$. 
procedure (e.g., perturbative coupling, chiral, $1 / N_{C}$ etc.) with which to control the one-loop approximation, and (b) the omission of potentially important short-distance contributions (e.g., the chiral counterterms $\left(b_{a}, c_{a}\right), t$-channel resonances, etc.).

2. Vector meson dominance. Pure vector meson dominance models, such as the three pole model of Ref. [16], omit all non-resonant Goldstone boson continuum contributions. This practice is not justified by any deep theoretical arguments, but rather by one's experience in the isoscalar channel where an acceptable $\chi^{2}$ is obtained with a three-pole only fit. The one-loop heavy baryon calculation implies, however, that the continuum contributions need not be negligible in the strangeness sector. Moreover, the prediction of Ref. [16] relies on questionable assumptions about the asymptotic behavior of the form factors. The hybrid model of Refs. [19,20] attempts to model both resonant and non-resonant kaon cloud contributions in a self-consistent manner while avoiding the problematic assumptions regarding asymptopia. In treating the two kaon continuum, however, the hybrid model still invokes hadronic meson-baryon form factors to cut-off the loop integrals form momenta above $\Lambda_{\chi}$. Consequently, this approach contains the same ambiguities discussed in the preceeding paragraphs.

3. Constituent quarks. Models wherein the nucleon strangeness moments arise from the strangeness "content" of consitutent quarks can be challenged on three fronts: (a) the credibility of the model itself for the problem at hand; (b) the procedure for computing the constituent quark strangeness vector current; and (c) the proper treatment of collective or many-quark contributions. The chiral quark model calculation illustrates all three issues. As discussed earlier, the problem of double counting the lightest pseudscalar states raises questions about the naïve chiral quark model. Although this difficutly can be addressed using more sophisticated treatments [73], the second issue is more problematic. As in the case of baryon effective theories, the calculation of the constituent quark strangeness matrix elements employs loops. A consistent chiral quark calculation implies the presence of terms in the chiral quark Lagrangian which carry unknown coefficients. These coefficients cannot be determined without knowing the strangeness matrix elements themselves. This dilemma is the same one which hampers heavy baryon CHPT as a predictive tool. As a fall back, one can employ form factors to cut off the loop integrals at a scale $\Lambda_{\chi}$ as we did in arriving at the numbers in Table I, but price one pays is the presence of all the ambiguities encountered when using hadronic form factors in hadronic loops. Presumably, the spread in predictions for the constituent quark strangeness currents is as broad as are the kaon cloud dominance predictions for the nucleon's strangeness moments. When one considers models other than the chiral quark model, the situation is even less controlled. For example, the NJL model, which provides an alternative model for the constituent quark strangeness radius, gives a value for $\rho_{S}^{s}$ that has the opposite sign from the chiral quark prediction and about $40 \%$ of the magnitude.

Finally, when one compares the predictions for chiral quark model and non-linear $\sigma$-model predictions for $\rho_{D}^{s}$, one finds that the former is a factor of five larger than the latter. In both cases, the form for the meson-fermion vertex is the same, including the approximate value of the form factor cut-off. One faces the question as to whether these two calculations give independent contributions which should be added, or whether there is some overlap between the two. One might argue in favor of the second possibility by noting that at the quark level, 
a loop involving a kaon and strange baryon intermediate state contains diagrams in which a constituent quark fluctuates into a freely propagating $S$-quark and kaon plus others in which the $S$-quark interacts with the other intermediate-state constituent quarks. In this line of reasoning, the chiral quark model calculation would give an over-prediction for $\rho_{D}^{s}$ due to the omission of quark-quark interactions (many-quark effects).

Experimental Implications. With these caveats in mind, it is interesting to ask what the up-coming neutral current experiments will be able to say about any of the model predictions in Table I. To that end, some observations about their magnitude and sign is in order. In the case of $\mu^{s}$, all of the models, except for the resonance saturation model, give $\mu^{s} \sim-\mu^{T=0}$. The large positive value for $\mu^{s}$ in the resonance saturation model results from a large loop contribution - enhanced by a factor of $\pi\left(m_{K} / \Lambda_{\chi}\right)$ - and the questionable absence of correspondingly large vector meson terms. As for the strangeness radius, all of the calculations containing kaon loops (except resonance saturation) give the same sign for $\rho^{s}$ and magnitudes which vary by a factor of five. The sign corresponds to the naïve expectation derived from the kaon cloud picture in which the kaon, containing the $\bar{s}$-quark, lives farther, on average, from the nucleon's center of mass than does the $\Lambda$, where the $s$-quark resides. The pole and resonance saturation models, however, give strangeness radii having much larger magnitudes and the opposite sign. The latter results follow from the fits of Refs. [57.58] which yield a large $\phi N N$ coupling. Note that in the case of the resonance saturation model, the large $\phi$-pole contribution to $\rho_{D}^{s}$ is cancelled to some extent by the continuum term (loops), whereas in the pure pole model, the $\phi$ contribution is cancelled to an even greater degree by the questionable $V^{\prime}$ residue. An experimental result consistent with resonance saturation value $\rho_{D}^{s}$ would suggest that resonant $t$-channel kaon and multi-pion rescattering (poles) are the most important physics behind the strangeneness radius. A significantly smaller result, or one having the opposite sign, would imply the presence of large continuum contributions going beyond one-loop order or important higher-mass resonance terms.

How might the various parity-violating electron scattering experiments do in terms of sorting out among these scenarios? The SAMPLE experiment at MIT-Bates [2].3] and " $G^{0}$ " experiment planned for CEBAF [四] anticipate a determination of $\mu^{s}$ with an error bar of \pm 0.2 . At this level of precision, these experiments could confirm the presence of a large strangeness magnetic moment (on the order of the resonance saturation prediction) or rule out the remaining predictions. It would be difficult for the SAMPLE and $G^{0}$ measurements to confirm any of these remaining predictions without significantly better precision. As far as the strangeness radius is concerned, one anticipates a determination of $\rho_{S}^{s}$ with an error of $\approx \pm 1.0$ from the Hall $\mathrm{A}$ and $\mathrm{C}$ experiments at CEBAF [4,60. These experiments could see a strangeness radius at the level of the pole and resonance saturation predictions and, at best could rule out (but not confirm) the remaining entries in Table I.

Parenthetically, one might note that forward angle parity-violating electron scattering experiments with a proton target [4, 6, [] are sensitive to the linear combination $\rho_{S}^{s}+\mu^{p} \mu^{s}$ where $\mu^{p} \approx 2.79$ is the proton's magentic moment [1, 10]. It has been suggested that a determination of this linear combination is useful as a "first pass" probe of the nucleon's strangeness vector current. Naïvely, a one might conclude that a small result for this quantity would indicate small magnitudes for $G_{E}^{(s)}$ and $G_{M}^{(s)}$. In the case of the resonance saturation model, for example, prediction for $\rho_{S}^{s}+\mu^{p} \mu^{s}$ is an order of magnitude smaller than the 
predicted values of either $\rho_{S}^{s}$ or $\mu^{s}$ alone, owing to a cancellation between the two terms. Similarly serious cancellations occur in the case of several of the other model predictions. One ought to be cautious, therefore, about drawing strong conclusions from a forward angle measurement alone. A pair of forward and backward angle measurements, allowing for separate determinations of $\mu^{s}$ and $\rho^{s}$, would be more relevant to the comparison of model predictions.

\section{SUMMARY}

In this study, we sought to delineate the extent to which chiral symmetry can be used to arrive at predictions for the nucleon's strangeness vector current form factors. Since CHPT has proven quite useful in other contexts, it is timely to analyze its usefulness in the case of nucleon strangeness. Moreover, since the role of the $s \bar{s}$ sea in the nucleon's low-energy properties is of considerable interest to the hadron structure community, and since significant experimental effort is being devoted to measuring the nucleon's strange quark form factors, one would like to possess an effective theory framework in which to understand the strong interaction dynamics behind the numbers to be extracted. Ideally, CHPT would have provided such a framework. We hope to have convinced the reader that nucleon strangeness (or, equivalently, the $\mathrm{SU}(3)$-singlet channel) presents barriers to the applicability of chiral symmetry not present in other cases where symmetry has proven more useful. To reiterate: the reason for this difficulty is that the quantity one wishes to predict - the strangeness (or SU(3)-singlet) vector current matrix element - is the same quantity one needs to know in order to make a prediction.

Consequently, we turned our attention to chiral models. We explored three such model approaches as a representative sampling: a resonance saturation model for the unknown lowenergy constants arising in CHPT; kaon cloud dominance models; and models in which chiral symmetry is used to obtain the strangeness currents of constituent $U$ - and $D$-quarks. These different approaches yield a rather broad range of predictions for the nucleon's strangeness radius and magnetic moment. This situation is not surprising, since none of the approaches relies solely on the underlying symmetries of low-energy QCD. We have tried to argue that there may exist one case in which a chiral model gives a credible prediction: the resonance saturation value for the Dirac strangeness radius. Of the models considered here, resonance saturation stays closest to the framework of CHPT while relying on well-defined phenomenological input. Moreover, it is clear which physics has not been included (higher-mass poles as well as non-resonant kaon rescattering and multi-pion continuum contributions). Should the experimental result for $\rho_{D}^{s}$ differ significantly from the resonance saturation value, one has a reasonable idea of what physics is likely to be responsible for the discrepancy. In all other cases, including the resonance saturation prediction for $\mu^{s}$, we have pointed out what appear to be good reasons to question the believability of chiral model calculations.

Nucleon strangeness remains a highly interesting subject on which experiment will shed some light. Assuming that the strangeness radius and magnetic moment are separately determined with the precision anticipated for the parity-violation experiments [2] [7, one could, for example, test the resonance saturation prediction for $\rho_{D}^{s}$. Given the ambiguities present

in the other approaches, an exprimental result for $\rho_{D}^{s}$ consistent with the corresponding pre- 
dictions would not be conclusive. A similar statement applies to all of the model predictions for $\mu^{s}$. In short, need for a better theoretical understanding of the nucleon's strangeness form factors remains an open problem. In this respect, a more detailed analysis within the context of dispersion relations appears to be a promising direction. In particular, one ought to look more carefully at contributions from non-resonant kaon rescattering and multi-pion intermediate states which are not included in the chiral model approaches discussed here.

\section{Acknowledgements}

We wish to thank J.L. Goity, R.L. Jaffe, X. Ji, D. Leinweber, U. Meissner, and N. Isgur for useful discussions and T. Schaefer for raising the question about the resonance saturation

model for the chiral counterterms. We also thank M. Frank for a critical reading of the manuscript. This work is supported in part by funds provided by the U. S. Department of Energy (D.O.E.) under contracts \#DE-AC05-84ER40150, \#DE-FG06-90ER40561, and \#DE-FG02-95-ER40907. M.J.M. was also supported by the National Science Foundation National Young Investigator Program. 


\section{REFERENCES}

[1] M.J. Musolf et al., Phys. Rep. 239, 1 (1994).

[2] MIT-Bates proposal \# 89-06 (1989), R.D. McKeown and D.H. Beck spokespersons.

[3] MIT-Bates proposal \#94-11 (1994), M. Pitt and E.J. Beise, spokespersons.

[4] CEBAF proposal \# PR-91-017 (1991), D.H. Beck, spokesperson.

[5] CEBAF proposal \# PR-91-004 (1991), E.J. Beise, spokesperson.

[6] CEBAF proposal \# PR-91-010 (1991), M. Finn and P.A. Souder, spokespersons.

[7] Mainz proposal A4/1-93 (1993), D. von Harrach, spokesperson.

[8] See, e.g., R. McKeown and M.J. Musolf in Symmetries and Fundamental Interactions in Nuclei W.C. Haxton and E.M. Henley (eds), World Scientific, Singapore (1995) and references therein.

[9] Los Alamos proposal \# 1173, W.C. Louis, spokesperson.

[10] M.J. Musolf and T.W. Donnelly, Nucl. Phys. A546 , 509 (1992); 550, 564(E) (1992).

[11] G.T. Garvey et al, Phys. Lett. B289 , 249 (1992); Phys. Rev. C48 , 1919 (1993).

[12] C.J. Horowitz et al., Phys. Rev. C48 , 3078 (1993).

[13] M.B. Barbaro et al., INT Preprint INT/DOE/ER/ 40561-243-INT96-00-112 (1996).

[14] K.-F. Liu, U. of Kentucky preprint UK/95-11 (1995) and references therein.

[15] D.B. Leinweber, Phys. Rev. D53 , 5115 (1996).

[16] R.L. Jaffe, Phys. Lett. B229 , 275 (1989).

[17] M.J. Musolf and M. Burkardt, Z. für Phys. C61 , 433 (1994).

[18] W. Koepf, S.J. Pollock, and E.M. Henley, Phys. Lett. B288 , 11 (1992); W. Koepf and E.M. Henley, Phys. Rev. C49 , 2219 (1994).

[19] H. Forkel et al., Phys. Rev. C50 , 3108 (1994).

[20] T. Cohen, H. Forkel, and M. Nielsen, Phys. Lett. B316 , 1 (1993).

[21] N.W. Park, J. Schechter, and H. Wiegel, Phys. Rev. D43, 869 (1991).

[22] S.-T. Hong and B.-Y. Park, Nucl. Phys. A561, 525 (1993).

[23] S.C. Phatak and S. Sahu, Phys. Lett. B321 , 11 (1994).

[24] H. Ito, Phys. Rev. C52, R1750 (1995).

[25] P. Geiger and N. Isgur, CEBAF Preprint \# TH-96-08 (1996).

[26] T.P. Cheng, Phys. Rev. D13 , 2161 (1976); J. Gasser, H. Leutwyler, and M.E. Sainio, Phys. Lett. B168 , 105 (1986).

[27] J. Ashman et al. (EMC), Nucl. Phys. B328 , 1 (1989).

[28] P.L. Anthony et al (E142), Phys. Rev. Lett. 71 , 959 (1993).

[29] B. Adeva et al. (SMC), Phys. Lett. B302, 533 (1993).

[30] D. Adams et al. (SMC), Phys. Lett. B329 , 399 (1994).

[31] K. Abe et al. (E143), Phys. Rev. Lett. 74 , 346 (1995).

[32] J. Gasser, H. Leutwyler, and M.E. Sainio, Phys. Lett. B253 , 252 (1991).

[33] E. Jenkins and A. Manohar, Phys. Lett. B255 , 558 (1991); Phys. Lett. B259 , (1991).

[34] J. Dai, R. Dashen, E. Jenkins, and A. Manohar, UCSD Preprint PTH 94-19 (1995).

[35] B. Ehrnsperger and A. Schafer, UFTP Preprint 377-1994 (1994).

[36] J. Lichtenstadt and H. Lipkin, Tel Aviv Preprint TAUP-2244-95 (1995).

[37] See, e.g., S. Capstick, "Constituent Quark Models of Baryon Structure and Strong Decays", and "A Comparison with Experimental Results, and Outstanding Issues in Baryon Physics", in Proceedings of the NATO Advanced Study Institute on Hadron 
Spectroscopy and the Confinement Problem, D.V. Bugg, Ed., July 1995, University of London and the University of Swansea.

[38] CCFR, A.O. Bazarko, et al., Z. für Phys. C65 , 189 (1995); CCFR, S.A. Rabinowitz, et al., Phys. Rev. Lett. 70 , 134 (1993).

[39] We are grateful to J.W. Negele for clarifying this issue for us.

[40] J. Donoghue, E. Golowich, and B. Holstein, Dynamics of the Standard Model, Cambridge University Press, Cambridge (1992).

[41] Proceedings of the Workshop on Effective Theories of the Standard Model, U.-G. Meissner (ed.), World Scientific, Singapore (1992).

[42] E. Jenkins et al., Phys. Lett. B302 , 482 (1993).

[43] M. N. Butler, M. J. Savage, R. P. Springer, Phys. Rev. D49 , 3459 (1994).

[44] M. K. Banerjee and J. Milana, Univ. of Maryland preprint UMPP \#96-19 (1995), hepph/9508340.

[45] T. D. Cohen, Phys. Lett. B359 , 23 (1995).

[46] H. Georgi, Weak Interactions and Modern Particle Theory, Benjamin/Cummings, Menlo Park (1984), Chapters 5-6.

[47] A. Manohar and H. Georgi, Nucl. Phys. B234, 189 (1984).

[48] R.G. Sachs, Phys. Ref. 126, 2256 (1962).

[49] In Ref. [42], a normalization to $m_{N}$ rather than $\Lambda_{\chi}$ was employed for the EM anomalous magnetic moment Lagrangian and the notation $\mu_{D}$ and $\mu_{F}$, rather than $b_{+}$and $b_{-}$, was used for the coefficients of the commutator and anti-commutator terms, respectively.

[50] M. J. Musolf, to be published.

[51] In the analysis of Ref. [42], a fit to the entire baryon octet was performed. For illustrative purposes, however, we need consider only the nucleons. In the case of the charge radii, there exist no measurements for the strangeness $\neq 0$ octet baryons.

[52] P. Federbush, M.L. Goldberger, and S.B. Treiman, Phys. Rev. 112, 642 (1958).

[53] G. Ecker et al., Phys. Lett. B223 , 425 (1989).

[54] G. Ecker et al., Nucl. Phys. B321 , 311 (1989).

[55] J.J. Sakurai, Currents and Mesons, University of Chicago Press, Chicago (1969).

[56] B. Holzenkamp, K. Holinde, and J. Speth, Nucl. Phys. A500 , 485 (1989).

[57] H. Genz and G. Höhler, Phys. Lett. B61 , 389 (1976).

[58] G. Höhler et al., Nucl. Phys. B114, 505 (1976).

[59] G. Höhler and E. Pietarinen, Nucl. Phys. B95 , 210 (1975) and references therein.

[60] P. Mergell, U.-G. Meissner, and D. Drechsel, Nucl. Phys. A596 , 367 (1996).

[61] J. Gasser, M.E. Sainio, A. Svarc, Nucl. Phys. B307 , 779 (1988).

[62] H.A. Bethe and F. deHoffman, Mesons and Fields V. II, Row and Peterson, Evanston (1955).

[63] T. Hatsuda, Phys. Rev. Lett. 65 , 543 (1990).

[64] T. Meissner, PLB340, 226 (1994).

[65] D.B. Kaplan and A. Manohar, Nucl. Phys. B310 , 527 (1988).

[66] Y. Nambu and G. Jona-Lasinio, Phys. Rev. 124, 246, 255 (1961).

[67] One may wish to compare the expression in Eq. (50) with the magnetic moment term in the chiral quark Lagrangian of Ref. [47. The latter contains an additional factor of $m / \Lambda_{\chi}$ in order to make explicit the chirality-violating character of the magnetic moment interaction. We choose instead a normalization which goes over to the form of Eq. (15) in 
the heavy quark limit. For simplicity, we have also ignored isospin-violating corrections which break the constituent quark EM magnetic moment relation $\mu_{U}=-2 \mu_{D}$.

[68] P.L. Chung and F. Coester, Phys. Rev. D44, 229 (1991).

[69] S.J. Brodsky and F. Schlumpf, Phys. Lett. B329 , 111 (1994).

[70] I.G. Aznauryan, A.S. Bagdasaryan, and N.L. Ter-Isaakyan, Yad. Fiz.36, 1278 (1982).

[71] N. Isgur, CEBAF preprint \# TH-92-31 (1992).

[72] N. Isgur, private communication.

[73] M. R. Frank, P. C. Tandy, and G. Fai, Phys. Rev. C43 , 2808 (1991). 


\section{FIGURES}

FIG. 1. One kaon loop contributions to strangeness vector current form factors of a non-strange fermion $f$ (nucleon or constituent quark). Here, $\times$ denotes insertion of the current $\bar{s} \gamma_{\mu} s$ and $f^{\prime}$ denotes a strangeness +1 fermion (e.g. $\Lambda$ or consituent $S$-quark.

FIG. 2. Resonance contribution to nucleon vector current form factors. Here $V$ denotes a vector meson and $\times$ denotes a vector current $(\mathrm{EM}$, strangeness, baryon number, etc.).

FIG. 3. Chiral quark model for nucleon strangeness. Shaded circle represents strange-quark vector current matrix element of a constituent $U$ - or $D$-quark, generated by the processes shown in Fig. 1.

FIG. 4. Nucleon strangeness vector current moments in the non-linear $\sigma$-model with hadronic form factors. Dimensionless Dirac strangeness radius (panel (a)) and strangeness magnetic moment (panel (b)) are shown as functions of the form factor cut-off parameter. To set the scale, note that the nucleon's dimensionless isovector EM Dirac radius is $\rho_{D}^{T=1}=-4.68$. 
This figure "fig1-1.png" is available in "png" format from: http://arxiv.org/ps/nucl-th/9607021v1 
This figure "fig2-1.png" is available in "png" format from: http://arxiv.org/ps/nucl-th/9607021v1 
This figure "fig1-2.png" is available in "png" format from: http://arxiv.org/ps/nucl-th/9607021v1 
This figure "fig1-3.png" is available in "png" format from: http://arxiv.org/ps/nucl-th/9607021v1 